\title{
The performance prediction and optimization of the fiber-reinforced composite structure with uncertain parameters
}

\author{
X.Y. Cui ${ }^{1,2, *}$, X.B. Hu ${ }^{1,2}$, Z.M. Liang ${ }^{1,2}$, G.Y. Li $^{1,2}$ \\ ${ }^{1}$ State Key Laboratory of Advanced Design and Manufacturing for Vehicle Body, Hunan \\ University, Changsha, 410082, PR China \\ ${ }^{2}$ Joint Center for Intelligent New Energy Vehicle, Shanghai, 201804, PR China
}

\begin{abstract}
The fiber-reinforced composites display the random fiber orientations and uncertain material parameters because of the manufacturing error and scattering feature. For this problem, the uncertain prediction and optimization based on the transformed perturbation stochastic method, the edged-based smoothing technique and the optimization theory is presented. In this method, the non-Gaussian probability density functions and the cumulative distribution functions of multi-variables for stochastic static responses of fiber-reinforced composite structure are explicitly exhibited as the prediction result compared with the Monte Carlo solution. Unlike the direct MCs, it only needs an iteration as the FEM and has the potential in the complex construction.
\end{abstract} In order to improve the efficiency and capability to resist the mesh distortion compared with the traditional stochastic FEM, the edged-based smoothing technique is introduced into the present framework. Furthermore, the stable performance feature of the fiber-reinforced composite in the uncertain working condition is presented. Consequently, objectives of the structural stability and insensitivity criteria based on the second-order perturbation expansion are proposed, and overall uncertain conditions coupled by uncertain fiber orientation, external load, material parameters and geometry sizes are analyzed and optimized within this framework, respectively.

\footnotetext{
* Corresponding author. Tel: 86-731-8821717; Fax: 86-731-8822051

Email address: cuixy@hnu.edu.cn (X.Y. Cui)
} 
To verify the effectiveness and accuracy of the uncertain analysis and optimization in this study, three examples including the composite plates, the composite shell and the composite top cover of automobile are provided.

Key words: The uncertain prediction and optimization; edged-based smoothing technique; fiber-reinforced composite structure; transformed perturbation stochastic method. 


\section{Introduction}

Advanced composites have some superior characters, such as light weight, high strength, good anti-fatigue feature and environment resistance. As a consequence, it has been applied into aviation, aerospace, shipbuilding and automobile industry. In practical applications, material properties of the composite laminated plates and shells are usually subjected to a certain amount of scattering [1]. Furthermore, the current manufacture technology can only control the certainty of the laminated material parameters within $20 \%$ and the stochastic character of measurement procedure should also been considered. In addition, there exist the tolerance and the stochastic angle uncertainty of technology when laying fibers on each layer. Besides, geometry sizes, external loads and environment conditions are also uncertain variables which affect the normal use of laminated plates and shells. Therefore, it is reasonable to treat materials, fiber orientations, geometry sizes and external loads as stochastic parameters and formulate the uncertain analysis, especially in the precise reliable problems.

Composite structures are overdesigned due to a lack of reliable predictive methods for the complicatedly uncertain structure. As a consequence, many researches $[2,3]$ based on the probabilistic predication are recently presented. M.F. Ngah [4] proposed the performance prediction of composite structures based on the spectral stochastic finite element method (SSFEM). Souvik Chakraborty et.al [5] proposed the polynomial correlated function expansion (PCFE) to predict stochastic 
free vibration analysis using the 1200 samples compared with MCs and N. Carrere [6] proposed the efficient structural computations to analyze composite applications with parameter uncertainty. The stochastic analysis of composite structures based on material microstructure is also implemented by George Stefanou et.al [7], M.M.S. Dwaikat [8], and T.S. Mesogitis [9]. The study about predication in vibrational behavior of the long fiber-reinforced composites under uncertainty in fiber orientations is presented by K.Sepahvand [10]. However, it is still essential and vital to introduce the efficient method in prediction based on the feasible probability theory to visually and explicitly exhibit the probability density and cumulative functions of multi-variables instead of MCs which needs a large amount of computational cost. In this paper, a unified framework [11] integrating the classical perturbation stochastic finite element method (PSFEM) and the change-of-variable technique together is taken into the macroscopic structure predication of fiber-reinforced composite, and consequently the probability density and cumulative functions of multi-variables of Gaussian and non-Gaussian can be calculated. Unlike the direct MCs, it only needs an iteration as the FEM and has the potential in the complex construction, especially into the large-scale problems. These existing uncertainties can be defined as random variables whose probability density functions can be determined unambiguously based on the statistical analysis of experimental or sample data. The classic perturbation stochastic method (PSFEM) [12-14] and the spectral stochastic method (SSFEM) $[15,16]$ are two main branches of the stochastic finite element (SFEM) [17-19]. In perturbation stochastic finite element method, the random system matrix 
and loading vector are expanded by the Taylor series. Due to the disproportional increase of the computational cost, the first order approximation is the most feasible strategy [20]. Eventually, response formulas of the stochastic structure are translated into linear functions of random variables based on the first order Taylor expansion. Stochastic perturbation method has been significantly developed these years. Kaminski [21] et al. applied the generalized stochastic perturbation method to thermal stresses and deformation analysis of spatial steel structures. Recently, the dual iterative stochastic perturbation-based finite element was also proposed by Kamiński [22] to replace the linearization procedure with the iterative one. Li et al. [23] applied the mean-centered first order perturbation technique to compute the second-order statistics (mean and standard deviation) of the critical thermal buckling temperature and an efficient $\mathrm{C} 0$ stochastic finite element based on the first-order perturbation technique (FOPT) is proposed by Mohammad Talha et al. [24].

The high-precise determination of reliable problems for the perturbation method based on the first order Taylor expansion is still an open problem in computational mechanics. Reference [21] emphasize that the usage and precision strongly rely on the input random field types, their correlations as well as numerical technique used to solve the basic deterministic problem. It obviously indicates that there are two major approaches to obtain the high precise results: the higher orders expansion technology and the basic deterministically high-precision numerical method. To improve the accuracy of the results, Marcin Kamiński [26,27] presented the generalized $n$th order stochastic perturbation technique to test the procedure on simple problems with 
analytical solutions sacrificing a plenty of computational cost. Though the higher order stochastic perturbation technique is employed to get the fine result, the potential defect of low accuracy in traditional FEM occurs to greatly influence and even eliminate the high accuracy which brought by the $n$th order technique. For the purpose of significantly improving accuracy without increasing computational cost, it is necessary to introduce the high efficient numerical method instead of the finite element method (FEM). In some practical occasions especially in complex structures, the result of FEM is seriously deteriorated by the easily-distorted mesh. In other words, it is the fatal limitation that the result heavily relies on the quality of mesh. Thus it is much better that the numerical method should have the capability to resist the mesh distortion.

Many modified numerical methods have appeared to amend the shortcomings of FEM. The conforming radial point interpolation method was introduced into the static and free vibration analysis of laminated composite plates by Zhao et al. [28]. Liu et al. [29] applied the cell-based smoothed finite element method (CS-FEM) and the generalized nth order stochastic perturbation technology into stochastic problems. This method can improve the accuracy significantly for stochastic problem. However, the determined method of CS-FEM is more suitable to the regular mesh and the accuracy also has potentials to improve further. Concerning the determined method, Liu [30] developed the edge-based smoothed finite element method (ES-FEM) formulation and made a major contribution to expand the use of the ES-FEM in various application fields, for instance, fluid-structure interaction [31], crack problems 
[32,33], acoustic problems [34] etc. ES-FEM has its advantage that it is insensitive to the mesh distortion, moreover, it just uses the triangle elements to get highly accurate solutions without costing extra time, which is much more efficient than traditionally stochastic FEM. Recently, ES-FEM has been applied into laminated plates by Cui [35] and CAD/CAE integrated system by Wang et.al [36]. It has been gradually proven an accurate and reliable tool.

In macroscopic mechanical properties analysis, fiber composites are modeled as anisotropic materials. Therefore, the anisotropic material optimization [37-39] lays the foundation for composite optimization. In stiffness laminate design, the fiber orientation angles are usually chosen as the design variables. According to the macroscopic mechanical properties analysis of fiber composite, the variation of fiber orientation angle can be treated as the modification of material property matrix. Many research works have been carried out to optimize composite laminated plates to obtain a maximum stiffness or strength. Kim et al. [40] investigated the optimal stacking sequence of a symmetrical laminated plate under in-plane loading to maximize the load bearing ability. Park [41] also investigated a laminated plate under in-plane loading and gave an optimal design method. Kam and Chang [42] used the multi-start global optimization technique to optimize the ply orientation of a laminated plate for a maximum stiffness. Tauchert and Adibhalta [43] optimized the arrangement of a composite laminated plate for a maximum bending stiffness. Refs [44-46] studied the multilevel optimization of the laminated plates in which the ply orientation and thickness were designed at each level of optimization. In these methods, the material 
property of the laminated plate was always regarded as deterministic and whereby the design process was formulated as a deterministic optimization problem. Thereafter, some studies on the uncertain optimization are gradually appeared. Jiang [47] proposed the uncertain optimization of composite plates based on the interval model. The probabilistic analysis and optimization of nanofiber-enhanced composite are presented by M. Rouhi et.al $[48,49]$. In this paper, the optimization of fiber-reinforced composite based on the transformed perturbation stochastic method and edged-based smoothing element method is proposed. In most engineering cases, the structure stability and the maximum stiffness character in complex conditions simultaneously coupled by uncertain angle orientations, external loads, material parameters and geometry sizes, are the hotspot to be studied. The objectives in the stability and sensibility criteria based on the second-order perturbation expansion are proposed and the overall uncertain condition coupled by various variables is analyzed using this strategy. The initial and optimization results are visually illustrated by the probability density function and cumulative distribution functions.

The organization of this paper is listed as follows: In Section 2, the basic equations of composite plates and shells are briefly formulated, and the strain smoothing technique and distinctly superior features of edged-based finite element method (ES-FEM) are also illustrated. The perturbation stochastic finite element method combining with mathematical theories based on the change-of-variable technique is discussed in Section 3. In section 4, the main object optimization method is considered and two optimization objectives are presented based on the perturbation 
method. Three numerical examples including the composite plates, the composite shell and the composite top cover of automobile are provided to verify the effectiveness and accuracy of the uncertain analysis and optimization in Section 5. In Section 6, some vital conclusions are given.

\section{Edge-based smoothing finite element method (ES-FEM) for the composite structure}

\subsection{Basic equation of the laminated structure}

A typical shell model is shown in Fig. 1. Based on the first-order shear deformation laminated theory, the displacement field can be expressed as:

$$
\begin{aligned}
& u=u_{0}+z \theta_{y} \\
& v=v_{0}-z \theta_{x} \\
& w=w_{0}
\end{aligned}
$$

Where $u_{0}, v_{0}$ and $w_{0}$ are the displacement of the mid-surface in the direction of $\mathrm{x}, \mathrm{y}, \mathrm{z}$. $\theta_{x}$ and $\theta_{y}$ are the rotation angles towards the $\mathrm{x}$-axis and y-axis, respectively.

The strain vector $\boldsymbol{\varepsilon}$ can be defined in terms of the mid-plane deformations:

$$
\boldsymbol{\varepsilon}=\left\{\begin{array}{c}
\varepsilon_{x x} \\
\varepsilon_{y y} \\
\gamma_{x y} \\
\gamma_{x z} \\
\gamma_{y z}
\end{array}\right\}=\left\{\begin{array}{c}
\frac{\partial u}{\partial x} \\
\frac{\partial v}{\partial y} \\
\frac{\partial u}{\partial x}+\frac{\partial v}{\partial y} \\
\frac{\partial u}{\partial z}+\frac{\partial w}{\partial x} \\
\frac{\partial v}{\partial z}+\frac{\partial w}{\partial y}
\end{array}\right\}=\left\{\begin{array}{c}
\boldsymbol{\varepsilon}_{\mathbf{m}} \\
\mathbf{0}_{2 \times 1}
\end{array}\right\}+z\left\{\begin{array}{c}
\boldsymbol{\varepsilon}_{\mathbf{b}} \\
\mathbf{0}_{2 \times 1}
\end{array}\right\}+\left\{\begin{array}{c}
\mathbf{0}_{3 \times 1} \\
\boldsymbol{\varepsilon}_{\mathrm{s}}
\end{array}\right\}
$$

Where $\boldsymbol{\varepsilon}_{\mathbf{m}}$ stands for the membrane strain, $\boldsymbol{\varepsilon}_{b}$ is the bending strain and $\boldsymbol{\varepsilon}_{s}$ denotes the shear strain, which are given as the following way: 


$$
\boldsymbol{\varepsilon}_{\mathbf{m}}=\left\{\begin{array}{c}
\frac{\partial u_{0}}{\partial x} \\
\frac{\partial v_{0}}{\partial y} \\
\frac{\partial u_{0}}{\partial y}+\frac{\partial v_{0}}{\partial x}
\end{array}\right\} \boldsymbol{\varepsilon}_{\mathbf{b}}=\left\{\begin{array}{c}
\frac{\partial \theta_{x}}{\partial x} \\
-\frac{\partial \theta_{y}}{\partial y} \\
\frac{\partial \theta_{y}}{\partial y}-\frac{\partial \theta_{x}}{\partial x}
\end{array}\right\} \boldsymbol{\varepsilon}_{\mathbf{s}}=\left\{\begin{array}{l}
\frac{\partial w}{\partial x}+\theta_{y} \\
\frac{\partial w}{\partial y}-\theta_{x}
\end{array}\right\}
$$

The constitutive relationship of laminated plates and shells can be expressed as:

$$
\hat{\boldsymbol{\sigma}}=\hat{\mathbf{D}} \hat{\boldsymbol{\varepsilon}}
$$

Where

$$
\hat{\boldsymbol{\sigma}}=\left\{\begin{array}{lll}
\hat{\boldsymbol{\sigma}}_{m}^{T} & \hat{\boldsymbol{\sigma}}_{b}^{T} & \hat{\boldsymbol{\sigma}}_{s}^{T}
\end{array}\right\}^{T} \hat{\boldsymbol{\varepsilon}}=\left\{\begin{array}{lll}
\hat{\boldsymbol{\varepsilon}}_{m}^{T} & \hat{\boldsymbol{\varepsilon}}_{b}^{T} & \hat{\boldsymbol{\varepsilon}}_{s}^{T}
\end{array}\right\}^{T} \hat{\mathbf{D}}=\left[\begin{array}{ccc}
\mathbf{A} & \mathbf{B} & 0 \\
\mathbf{B} & \mathbf{D} & 0 \\
0 & 0 & \mathbf{S}
\end{array}\right]
$$

Where $\hat{\sigma}_{m}^{T}=\left\{N_{x}, N_{y}, N_{x y}\right\}^{T}$ is the force vector in the mid-surface, $\hat{\sigma}_{s}=\left\{Q_{x}, Q_{y}\right\}^{T}$ is the transverse shear force vector and $\hat{\sigma}_{b}^{T}=\left\{M_{x}, M_{y}, M_{x y}\right\}^{T}$ is the bending moment vector Besides, the membrane stiffness (A), the bending stiffness (D), the bending-extensional coupling stiffness $(\mathbf{B})$ and the transverse shear stiffness $(\mathbf{S})$ are respectively illustrated:

$$
\begin{gathered}
\mathbf{A}=\left[\begin{array}{lll}
A_{11} & A_{12} & A_{16} \\
A_{12} & A_{22} & A_{26} \\
A_{16} & A_{26} & A_{66}
\end{array}\right] \mathbf{B}=\left[\begin{array}{lll}
B_{11} & B_{12} & B_{16} \\
B_{12} & B_{22} & B_{26} \\
B_{16} & B_{26} & B_{66}
\end{array}\right] \\
\mathbf{D}=\left[\begin{array}{lll}
D_{11} & D_{12} & D_{16} \\
D_{12} & D_{22} & D_{26} \\
D_{16} & D_{26} & D_{66}
\end{array}\right] \mathbf{S}=\left[\begin{array}{cc}
\kappa_{1} S_{44} & \sqrt{\kappa_{1} \kappa_{2}} S_{45} \\
\sqrt{\kappa_{1} \kappa_{2}} S_{45} & \kappa_{2} S_{55}
\end{array}\right]
\end{gathered}
$$

In which $\kappa_{1}$ and $\kappa_{2}$ denote shear correction factors.

The various elements of stiffness matrixes in Eq. (6) are defined as: 


$$
\begin{aligned}
& A_{i j}=\sum_{k=1}^{N_{I}} \bar{Q}_{i j}^{(\mathrm{k})}\left(\mathrm{t}_{k}-\mathrm{t}_{k+1}\right) B_{i j}=\frac{1}{2} \sum_{k=1}^{N_{I}} \bar{Q}_{i j}^{(\mathrm{k})}\left(\mathrm{t}_{k}^{2}-\mathrm{t}_{k+1}^{2}\right) \\
& D_{i j}=\frac{1}{3} \sum_{k=1}^{N_{I}} \bar{Q}_{i j}^{(\mathrm{k})}\left(\mathrm{t}_{k}^{3}-\mathrm{t}_{k+1}^{3}\right) S_{i j}=\sum_{k=1}^{N_{I}} \bar{Q}_{i j}^{(\mathrm{k})}\left(\mathrm{t}_{k}-\mathrm{t}_{k+1}\right)
\end{aligned}
$$

Where subscripts $i$ and $j$ of $A_{i j}, B_{i j}$ and $D_{i j}$ are defined as: $i, j=1,2,6$, while subscripts concerning $S_{i j}$ are defined as: $i, j=4,5 . \mathrm{t}_{k}$ and $\mathrm{t}_{k+1}$ denote the distance from the reference mid-surface to the outer and inner surfaces of the $k$ th layer, respectively, which are shown in Fig. (2).

\subsection{Edge-based strain smoothing technique}

In the present method, the problem domain is discretized into triangular elements as the means of FEM. The whole domain $\Omega$ is divided into $N_{e}$ triangular elements with $N_{\text {edge }}$ edges. To perform a smoothing operation, a smoothing domain of each edge is constructed by sequentially connecting two corner points of each edge with centroids of surrounding triangle elements.

The displacement in problem domain is interpolated using the element shape functions, which are constructed as same as the way in the linear standard FEM. The generalized mid-plane displacements vector $\mathbf{u}=\left\{u_{o i}, v_{o i}, w_{i}, \theta_{x}, \theta_{y}\right\}^{T}$ can be expressed as :

$$
\mathbf{u}(\mathbf{x})=\sum_{i=1}^{3} \mathbf{N}_{i}(\mathbf{x}) \mathbf{d}_{i}
$$

For interior edges which shown in Fig. (3), the integration domain of edge $k$ is built by assembling two sub-domains which situated in two neighbor elements. On the other hand, the integration domain of the boundary edge is constructed by only one 
sub-domain of the corresponding element, as shown in Fig. (4). Then introducing the smoothing operation, the smoothed strain of the smoothing area $\Omega_{k}\left(\Omega_{k 1}+\Omega_{k 2}=\Omega_{k}\right)$ is given as:

$$
\bar{\varepsilon}_{k}=\frac{1}{A_{k}}\left(\int_{\Omega_{k 1}} \varepsilon_{k 1}(\mathrm{x}) \mathrm{d} \Omega+\int_{\Omega_{k 2}} \varepsilon_{k 2}(\mathrm{x}) \mathrm{d} \Omega\right)
$$

Where $A_{k}$ denotes the area of a smoothing domain, and $\varepsilon_{k 1}$ and $\varepsilon_{k 2}$ are compatible strains defined in elements $\Omega_{k 1}$ and $\Omega_{k 2}$, respectively.

Because of the linear shape function is used, the compatible strain is a constant in each smoothing sub-domain. As a consequence, the formula can be rewritten as:

$$
\begin{aligned}
\bar{\varepsilon}_{k} & =\frac{1}{A_{k}}\left(\varepsilon_{k 1} A_{k 1}+\varepsilon_{k 2} A_{k 2}\right) \\
& =\frac{A_{k 1}}{A_{k}} \mathbf{B}_{m}^{k 1}\left(\mathrm{x}_{c k 1}\right) \mathbf{d}_{k 1}+\frac{A_{k 2}}{A_{k}} \mathbf{B}_{m}^{k 2}\left(\mathrm{x}_{c k 2}\right) \mathbf{d}_{k 2}=\overline{\mathbf{B}}_{m k} \mathbf{d}_{k}
\end{aligned}
$$

Where $A_{k 1}$ and $A_{k 2}$ are areas of two neighbor sub-domains $\Omega_{k 1}$ and $\Omega_{k 2}, \mathbf{d}_{k 1}$ and $\mathbf{d}_{k 2}$ are the nodal displacement vectors of corresponding neighbor elements. $\mathbf{d}_{k}$ is the displacements of the nodes associated with the edge $k$, and $\mathbf{B}_{m}^{k l}\left(\mathrm{x}_{c k l}\right)$ is the compatible strain matrices of the smoothing sub-domain $\Omega_{k I}(I=1,2)$, which are given as:

$$
\mathbf{B}_{m}^{k I}\left(\mathrm{x}_{c k 1}\right)=\left[\begin{array}{lll}
B_{m 1}^{k I} & B_{m 2}^{k I} & B_{m 3}^{k I}
\end{array}\right], \quad(\mathrm{I}=1,2)
$$

Where

$$
\mathbf{B}_{m i}^{k I}=\left[\begin{array}{ccccc}
\frac{\partial N_{i}\left(\mathrm{x}_{c k I}\right)}{\partial x} & 0 & 0 & 0 & 0 \\
0 & \frac{\partial N_{i}\left(\mathrm{x}_{c k I}\right)}{\partial y} & 0 & 0 & 0 \\
\frac{\partial N_{i}\left(\mathrm{x}_{c k I}\right)}{\partial y} & \frac{\partial N_{i}\left(\mathrm{x}_{c k l}\right)}{\partial x} & 0 & 0 & 0
\end{array}\right], \quad(\mathrm{i}=1,2,3)
$$

Then the Eq. (10), the smoothed membrane strain matrix $\mathbf{B}_{m i}^{k I}$ can be rewritten as: 


$$
\overline{\mathbf{B}}_{m k}=\frac{A_{k 1}}{A_{k}} \mathbf{B}_{m}^{k 1}+\frac{A_{k 2}}{A_{k}} \mathbf{B}_{m}^{k 2}
$$

Similarly, the bending strain matrix $\overline{\mathbf{B}}_{b k}$ is described as follows:

$$
\overline{\mathbf{B}}_{b k}=\frac{A_{k 1}}{A_{k}} \mathbf{B}_{b}^{k 1}+\frac{A_{k 2}}{A_{k}} \mathbf{B}_{b}^{k 2}
$$

The smoothed bending strain can be expressed as:

$$
\overline{\boldsymbol{\varepsilon}}_{b k}=\overline{\mathbf{B}}_{b k} \mathbf{d}_{k}
$$

In order to eliminate the shear locking, the discrete shear gap (DSG) method [50] is used. In the same way, the smoothed shear strain $\overline{\boldsymbol{\varepsilon}}_{s k}$ of the integration domain $\Omega_{k}$ can be expressed as:

$$
\overline{\boldsymbol{\varepsilon}}_{s k}=\overline{\mathbf{B}}_{s k} \mathbf{d}_{k}
$$

Where $\overline{\mathbf{B}}_{s k}$ is assembled by $\mathbf{B}_{s}^{k 1}$ and $\mathbf{B}_{s}^{k 2}$ as follows:

$$
\overline{\mathbf{B}}_{s k}=\frac{A_{k 1}}{A_{k}} \mathbf{B}_{s}^{k 1}+\frac{A_{k 2}}{A_{k}} \mathbf{B}_{s}^{k 2}
$$

Then the smoothed generalized strain in the $k$ th smoothing domain is illustrated according to the Eqs. (10), (15) and (16):

$$
\overline{\hat{\boldsymbol{\varepsilon}}}=\left\{\begin{array}{lll}
\overline{\boldsymbol{\varepsilon}}_{m k}^{T} & \overline{\boldsymbol{\varepsilon}}_{b k}^{T} & \overline{\boldsymbol{\varepsilon}}_{s k}^{T}
\end{array}\right\}^{T}=\overline{\mathbf{B}}_{k} \mathbf{d}_{k}
$$

In which

$$
\overline{\mathbf{B}}_{k}=\left[\begin{array}{lll}
\overline{\mathbf{B}}_{m k}^{T} & \overline{\mathbf{B}}_{b k}^{T} & \overline{\mathbf{B}}_{s k}^{T}
\end{array}\right]^{T}
$$

\subsection{Distinctive features of the ES-FEM}

The ES-FEM formulation is implemented based on the triangle elements and a linear FEM interpolation. Rather than the FEM which calculating the strain within each element, the smoothed strains of ES-FEM are calculated by the smoothing 
integration domains formed on each edge. According to the initial researches, the favorable features can be concluded as following:

1. The stiffness of the discretized model is softer compared with FEM model, and it could get upper bound solutions with respect to FEM and exact solution.

2. The ES-FEM based on the triangular elements can produce more accurate solutions than the FEM results based on the quadrilateral elements.

3. The continuity of the trial and test functions can be relaxed, allowing us to use more methods in building shape functions.

4. The solution of ES-FEM is insensitive to the mesh distortion, and it lowers the limitation of mesh quality. It could continue to work efficiently, even when the mesh is overly distorted.

5. The discrete systems equations of ES-FEM are symmetrical and sparse, and have the same dimensions as the FEM does. Thus, it is identical compared with the FEM in terms of computational cost, whereas it produces more accurate solutions.

\section{Transformed perturbation stochastic finite element method (TPSFEM) for the stochastic structure}

\subsection{Perturbation analysis of stochastic structure}

The statically determined structure function is considered:

$$
\mathbf{K u}=\mathbf{F}
$$

Where $\mathbf{K}$ stands for the stiffness matrix of the structure, $\mathbf{F}$ is the external loading vector and $\mathbf{u}$ is the static response of displacement. 
In most cases, the parameters of geometry and material are imprecise and random caused by the effects of manufacturing/construction tolerances, uncertain environmental conditions, and the unpredictable loads. For purpose of getting reliable solutions, the stochastic character must be introduced into the specific formula.

Utilizing the perturbation stochastic method, the first order Taylor expansion of the random matrix $\mathbf{K}(\mathbf{x})$ and the random loading vector $\mathbf{F}(\mathbf{x})$ can be expanded as:

$$
\begin{gathered}
\mathbf{K}=\mathbf{K}_{0}+\left.\sum_{i=1}^{n} \frac{\partial \mathbf{K}}{\partial \alpha_{i}}\right|_{\alpha=\alpha^{e}}\left(\alpha_{i}-\alpha_{i}^{e}\right)=\mathbf{K}^{\mathbf{e}}+\Delta \mathbf{K} \\
\mathbf{F}=\mathbf{F}_{\mathbf{0}}+\left.\sum_{i=1}^{n} \frac{\partial \mathbf{F}}{\partial \alpha_{i}}\right|_{\alpha=\alpha^{e}}\left(\alpha_{i}-\alpha_{i}^{e}\right)=\mathbf{F}^{\mathbf{e}}+\Delta \mathbf{F}
\end{gathered}
$$

Where $\boldsymbol{\alpha}^{e}=\left\{\alpha_{1}^{e}, \alpha_{2}^{e}, \ldots, \alpha_{n}^{e}\right\}$ is the expectation of respective random variables; $\mathbf{K}^{\mathbf{e}}$ and $\mathbf{F}^{\mathbf{e}}$ are the expectation of stiffness and load matrix. $\Delta \mathbf{K}$ and $\Delta \mathbf{F}$ are the deviation value of $\mathbf{K}^{\mathrm{e}}$ and $\mathbf{F}^{\mathrm{e}}$, respectively.

Substituting Eq. (21) into Eq. (20), then Eq. (20) can be translated into:

$$
\mathbf{u}=\left(\mathbf{K}^{e}+\Delta \mathbf{K}\right)^{-1}\left(\mathbf{F}^{e}+\Delta \mathbf{F}\right)
$$

Based on the Neumann expansion, $\left(\mathbf{K}^{e}+\Delta \mathbf{K}\right)^{-1}$ can be expressed as:

$$
\left(\mathbf{K}^{e}+\Delta \mathbf{K}\right)^{-1}=\mathbf{K}^{e}+\sum_{k=1}^{\infty}\left(\mathbf{K}^{e}\right)^{-1}\left(-\Delta \mathbf{K}\left(\mathbf{K}^{e}\right)^{-1}\right)^{k}
$$

Then Eq. (23) is introduced into Eq. (22). Neglecting the higher orders, the function Eq. (22) can be simplified as following:

$$
\mathbf{u} \approx\left(\mathbf{K}^{e}\right)^{-1} \mathbf{F}^{e}+\left(\mathbf{K}^{e}\right)^{-1} \Delta \mathbf{F}-\left(\mathbf{K}^{e}\right)^{-1} \Delta \mathbf{K}\left(\mathbf{K}^{e}\right)^{-1} \mathbf{F}^{e}
$$

Expanding the $\Delta F$ and $\Delta K$, the formula of $\mathbf{u}$ can be expressed into:

$$
\mathbf{u} \approx\left(\mathbf{K}^{e}\right)^{-1} \mathbf{F}^{e}+\left(\mathbf{K}^{e}\right)^{-1}\left(\left.\sum_{i=1}^{n} \frac{\partial \mathbf{F}(x)}{\partial \alpha_{i}}\right|_{\alpha=\alpha^{e}}\left(\alpha_{i}-\alpha_{i}^{e}\right)\right)-\left(\mathbf{K}^{e}\right)^{-1}\left(\left.\sum_{i=1}^{n} \frac{\partial \mathbf{K}(x)}{\partial \alpha_{i}}\right|_{\alpha=\alpha^{e}}\left(\alpha_{i}-\alpha_{i}^{e}\right)\right)\left(\mathbf{K}^{e}\right)^{-1} \mathbf{F}^{e}
$$


To sum up, parameters $A_{i}$ are introduced into the Eq. (25) and the simpler form is defined as:

$$
\mathbf{u}=\mathbf{A}_{0}+\sum_{i=1}^{n} \mathbf{A}_{i} \alpha_{i}
$$

Where

$$
\mathbf{A}_{0}=\left(\mathbf{K}^{e}\right)^{-1} \mathbf{F}^{e}-\sum_{i=1}^{n}\left(\mathbf{K}^{e}\right)^{-1}\left(\left.\frac{\partial \mathbf{F}(\alpha)}{\partial \alpha_{i}}\right|_{\alpha=\alpha^{e}}-\left.\frac{\partial \mathbf{K}(\alpha)}{\partial \alpha_{i}}\right|_{\alpha=\alpha^{e}}\left(\mathbf{K}^{e}\right)^{-1} \mathbf{F}^{e}\right) \alpha_{i}^{e}
$$

And

$$
\mathbf{A}_{i}=\left(\mathbf{K}^{e}\right)^{-1}\left(\left.\frac{\partial \mathbf{F}(\alpha)}{\partial \alpha_{i}}\right|_{\alpha=\alpha^{e}}-\left.\frac{\partial \mathbf{K}(\alpha)}{\partial \alpha_{i}}\right|_{\alpha=\alpha^{e}}\left(\mathbf{K}^{e}\right)^{-1} \mathbf{F}^{e}\right), i=1, \ldots, n
$$

\subsection{Transformed technique}

Although the expectation and standard deviation are easy to obtain, unfortunately, the probability density and cumulative distribution of random static response vector are impossible to achieve unless all stochastic variables are obeyed to Gaussian random field. However, in most practical cases, the uncertain variables can't be modeled as the Gaussian distribution. On the contrary, various variables may obey to different probability distribution, especially for non-Gaussian models. In this section, a hybrid stochastic method is introduced to achieve the probability density function of non-Gaussian random response.

Eq. (26) can be rewritten in matrix way:

$$
\left\{\begin{array}{c}
u_{1} \\
\vdots \\
u_{j} \\
\vdots \\
u_{m}
\end{array}\right\}=\left\{\begin{array}{c}
A_{0,1} \\
\vdots \\
A_{0, j} \\
\vdots \\
A_{0, m}
\end{array}\right\}+\sum_{i=1}^{n}\left\{\begin{array}{c}
A_{\mathrm{i}, 1} \\
\vdots \\
A_{i, j} \\
\vdots \\
A_{\mathrm{i}, m}
\end{array}\right\} x_{i}
$$

According to the algorithm of the linear equation, the $j$ th degree freedom of $\mathbf{u}$ is 
calculated:

$$
u_{j}=A_{0, j}+\sum_{i=1}^{n} A_{i, j} \alpha_{i}, \quad j=1, \ldots, \mathrm{m}
$$

Step 1: Rewriting the Eq. (30) as:

$$
u_{j}=A_{0, j}+\sum_{i=1}^{n} y_{\mathrm{i}, j}
$$

Where $y_{1, j}=A_{i, j} \alpha_{i}(\mathrm{i}=1, \ldots, \mathrm{n})$.The probability density functions of $y_{1, j}$ can be expressed as:

$$
f_{y_{1, j}}\left(y_{1, j}\right)=\frac{1}{\left|A_{i, j}\right|} f_{\alpha_{i}}\left(\frac{y_{1, j}}{A_{i, j}}\right), \quad i=1.2, \ldots, n
$$

Step2: Imagine $Z_{1, j}=y_{1, j}+y_{2, j}$, Eq. (31) can be rewritten as:

$$
u=A_{0, j}+Z_{1, \mathrm{j}}+\sum_{i=3}^{n} y_{\mathrm{i}, j}
$$

Where the probability density function of the variable $Z_{1, j}$ is unknown, however, it can be calculated as:

$$
\begin{aligned}
f_{Z_{1, j}}\left(Z_{1, \mathrm{j}}\right) & =\int_{y_{1, j}=\underline{b}_{1}}^{\bar{b}_{1}} f_{y_{1, j}}\left(Y_{1, j}\right) f_{y_{2, j}}\left(Y_{2, j}\right) d y_{1, j} \\
& =\frac{1}{\left|A_{2, \mathrm{j}}\right|} \int_{\alpha_{2}=\underline{a}_{1}}^{\bar{a}_{1}} f_{\alpha_{1}}\left(\alpha_{1}\right) f_{\alpha_{2}}\left(\frac{Z_{1, \mathrm{j}}-A_{1, \mathrm{j}} \alpha_{1}}{A_{2, \mathrm{j}}}\right) d \alpha_{1}
\end{aligned}
$$

Where $\underline{b}_{i}$ and $\bar{b}_{i}$ are the lower and upper bounds of integrate domain of variable $y_{\mathrm{i}, j}$. $\underline{a}_{i}$ and $\bar{a}_{i}$ are the lower and upper bounds of the integration domain of the random variable $\alpha_{i}$.

Step 3: Assume $Z_{2, \mathrm{j}}=Z_{1, j}+y_{3, j}$, Eq. (33) can be rewritten as:

$$
u_{j}=A_{0, j}+Z_{2, j}+\sum_{i=4}^{n} y_{\mathrm{i}, j}
$$

Where the probability density function of the variable $Z_{2, j}$ is described as: 


$$
f_{Z_{2, j}}\left(Z_{2, j}\right)=\int_{\alpha_{3}=\underline{a}_{3}}^{\bar{a}_{3}} f_{\alpha_{3}}\left(\alpha_{3}\right) f_{Z_{1, j}}\left(Z_{2, j}-A_{3, j} \alpha_{3}\right) d \alpha_{3}
$$

Step 4: Similar to above steps, the probability density function of the specific random variable $Z_{r, j}(\mathrm{r}=2, \ldots, \mathrm{n}-2)$ can be expressed as:

$$
f_{Z_{r, j}}\left(Z_{\mathrm{r}, j}\right)=\int_{\alpha_{\mathrm{r}+1}=\underline{a}_{r+1}}^{\bar{a}_{r+1}} f_{\alpha_{r+1}}\left(\alpha_{r+1}\right) f_{Z_{\mathrm{r}-1, j}}\left(Z_{\mathrm{r}-1, j}-A_{\mathrm{r}+1, \mathrm{j}} \alpha_{\mathrm{r}+1}\right) d \alpha_{\mathrm{r}+1}
$$

Where $Z_{r, j}=Z_{\mathrm{r}-1, j}+y_{\mathrm{r}+1, j}$

Step 5: After obtaining the probability density function of the variable $Z_{\mathrm{n}-2, \mathrm{j}}$, the last step is to analyze the probability density function of variable $u_{j}$. The Eq. (35) can be translated as:

$$
u_{j}=A_{0, j}+Z_{\mathrm{n}-2, \mathrm{j}}+y_{\mathrm{n}, j}
$$

And the probability density function of variable $u_{j}$ is expressed as:

$$
f_{u_{j}}\left(u_{j}\right)=\int_{\underline{a}}^{\bar{a}} f_{x_{n}}\left(x_{n}\right) f_{Y_{n-2, j}}\left(u_{j}-A_{0, j}-A_{\mathrm{n}, j} x_{n}\right) d x_{n}
$$

Based on the probability density function of $u_{j}$, the corresponding cumulative distribution function $F_{u_{j}}\left(u_{j}\right)$ can be expressed as:

$$
F_{u_{j}}\left(u_{j}\right)=\int_{-\infty}^{u_{0}} f_{u_{j}}(\mathrm{t}) d t
$$

\section{Optimization formulation}

Considering the manufacturing uncertainty of fiber path, the linear fiber of laminated plates and shells may not obey the designable linear angles. As a result, it unavoidably leads to the unstable and random solution, and even unreliable results. Besides, many other inevitably uncertain factors severely affect common use of laminated structures, for instance geometry parameters, material parameters, external loads and even working environments. It is necessary to utilize optimization theory to 
redesign angles of linear fiber paths. The multi-objective problems based on the perturbation theory are considered into this work. With regard to the various destinations, the optimization objectives are certainly different. Due to concentrate on the model stiffness feature, the displacement fields, especially some specific key-points, are studied. In order to obtain the most stable solution and eliminate the influences brought by the uncertain variables as far as possible, the stable displacement function is constructed:

$$
\min \text { object }_{1}=\sum_{i=1}^{n} \sum_{j=1}^{n} \frac{\partial \mathbf{u}}{\partial \alpha_{i}} \frac{\partial \mathbf{u}}{\partial \alpha_{j}} \operatorname{Cov}\left(\alpha_{i} \alpha_{j}\right)
$$

Where $i, j$ are the numbers of stochastic variable, $\boldsymbol{u}$ is the displacement field, and else components are listed as follows:

$$
\frac{\partial \mathbf{u}}{\partial \alpha_{i}}=\mathbf{K}_{0}^{-1}\left(\frac{\partial \mathbf{F}}{\partial \alpha_{i}}-\mathbf{u}_{0} \frac{\partial \mathbf{K}}{\partial \alpha_{i}}\right)
$$

And

$$
\operatorname{Cov}\left(\alpha_{i} \alpha_{l}\right)=\int_{j=1}^{n} \int_{i=1}^{n}\left(\alpha_{i}-\alpha_{i}^{0}\right)\left(\alpha_{j}-\alpha_{j}^{0}\right) f\left(\alpha_{i}, \alpha_{j}\right) d \alpha_{i} d \alpha_{j}
$$

Where $\alpha_{i}^{0}$ and $\alpha_{j}^{0}$ are the expectations of $\alpha_{i}$ and $\alpha_{j}$, respectively.

The displacement of key-points based on the 2nd-Taylor expansion of perturbation technique is also expressed as:

$$
\min \text { object }_{2}=\mathbf{u}_{0}+\frac{1}{2} \sum_{i=1}^{n} \sum_{j=1}^{n} \frac{\partial^{2} \mathbf{u}}{\partial \alpha_{i} \partial \alpha_{j}} \operatorname{Cov}\left(\alpha_{i} \alpha_{j}\right)
$$

Where

$$
\frac{\partial^{2} \mathbf{u}}{\partial \alpha_{i} \partial \alpha_{j}}=\mathbf{K}_{0}^{-1}\left(\frac{\partial^{2} \mathbf{F}}{\partial \alpha_{i} \partial \alpha_{j}}-\mathbf{u}_{0} \frac{\partial^{2} \mathbf{K}}{\partial \alpha_{i} \partial \alpha_{j}}-\frac{\partial \mathbf{u}}{\partial \alpha_{i}} \frac{\partial \mathbf{K}}{\partial \alpha_{j}}-\frac{\partial \mathbf{K}}{\partial \alpha_{i}} \frac{\partial \mathbf{u}}{\partial \alpha_{j}}\right)
$$

And $\mathrm{u}_{0}$ is the determined solution of displacement field. 
In this paper, the main object method is introduced into the multi-objective problems. The object $_{2}$ denoting the level of stiffness feature is assumed as the additional objective, and object $_{1}$ denoting the stable extent of solution and capability to resist uncertain distortion is considered as the major objective. According to the main object method, the additional subject is considered as the constrained condition while object $_{1}$ is the only objective.

$$
\begin{gathered}
\min \text { object }_{1}=\sum_{i=1}^{n} \sum_{j=1}^{n} \frac{\partial \mathbf{u}}{\partial \alpha_{i}} \frac{\partial \mathbf{u}}{\partial \alpha_{j}} \operatorname{Cov}\left(\alpha_{i} \alpha_{j}\right) \\
\text { object }_{2}=\mathbf{u}_{0}+\frac{1}{2} \sum_{i=1}^{n} \sum_{j=1}^{n} \frac{\partial^{2} \mathbf{u}}{\partial \alpha_{i} \partial \alpha_{j}} \operatorname{Cov}\left(\alpha_{i} \alpha_{j}\right) \leq \mathbf{U}_{\text {Initial }}
\end{gathered}
$$

Where $\mathbf{U}_{\text {Initial }}$ is the initial displacement field.

$$
\begin{aligned}
& \text { Boundary }:-\pi \leq \theta_{1} \leq \pi \\
& \text { Boundary2: }-\pi \leq \theta_{2} \leq \pi
\end{aligned}
$$

Where $\theta_{1}$ and $\theta_{2}$ are optimization variables.

\section{Problems analysis}

\subsection{Composite plate with uncertain external loads and fiber orientations}

The fiber-reinforced composite plate is a typical engineering component and frequently used in the practical engineering area. Due to the complexity of working condition, in most cases, the external loads are difficult and impractical to deal as the exact values, and instead, it is more practical to define it as the interval and probability model. Therefore, the external pressure is dealt as the stochastic variable, and considered as the Gumbel distribution. Besides, the Gaussian distribution is introduced to describe the manufacturing uncertain of fiber orientation, shown in 
Table 1.

A composite plate with the size $L \times L$ is showed in Fig. 5 which subjected to a uniform surface pressure $P$ on the top. Other geometry and material parameters as [35] for the problem are: $E_{11}=3.0 \times 10^{7} \mathrm{psi}, \quad E_{22}=0.75 \times 10^{6} \mathrm{psi}, G_{12}=4.5 \times 10^{5} \mathrm{psi}$, $G_{23}=3.75 \times 10^{5} \mathrm{psi}, \quad G_{13}=4.5 \times 10^{5} \mathrm{psi}, \quad v_{12}=v_{13}=v_{23}=0.25, L=20 \mathrm{in}, \quad t=0.02 \mathrm{in}$ and $\mathrm{P}=100 \mathrm{~N}$. The number of fiber plies is 9 , the thickness of odd pile is $t / 10$ and others are t/8. The nine-layer plate is symmetrically laminated with [60/-60/60/-60/60/-60/60/-60/60]. In order to compare with the determined solution, the reference result is obtained by ABAQUS with S8R5 element using the 4961 nodes. The results are dealt as $w_{c} E_{22} t^{3} \times 10^{3} /\left(\mathrm{P} \times \mathrm{L}^{4}\right)$ and listed in the Table 2 .

Then the transformed perturbation stochastic method is employed to illustrate results of multi-variables of non-Gaussian distributions. To verify the accuracy of present method, it is compared with the reference solution from Monte Carlo method. The Monte Carlo algorithm is the most stable and reliable method to get the accurate results as the reference solution by sacrificing a large amount of time (at least 100000 iterations), especially for complex problems. In distinct contrast, the transformed perturbation stochastic method just needs an iteration of algorithm and saves plenty of time. As a consequence, it has the large potential and superiority in large-scale engineering problem.

In probability theory, both the probability density function and the cumulative distribution function can be used to estimate the failure probabilities and reliabilities which act as the role to optimize stochastic structure. The probability density 
functions and the cumulative distribution functions of the deflection of the key-point which calculated by MCM with 100,000 samples and the present method are both plotted in Fig.6. It can be seen from Fig. 6 that the probability density function of the deflection of key-point is non-Gaussian remarkably. This indicates the static response of the stochastic structure is non-Gaussian when some random variables are non-Gaussian variables. Therefore, it is unreliable or even wrong to approximate the responses of stochastic structures as Gaussian fields if some random variables are non-Gaussian variables. Moreover, it can be found from Fig. 6 that the probability density function and the cumulative distribution function of the deflection of key-point yielded by this strategy match the reference results obtained by MCM well. Thus, the accuracy of this strategy is effective, when used for predicting the probability density function and the cumulative distribution function of the static response of the stochastic structure whose analytical solution is hard to obtain.

Then the uncertain optimization of symmetric fiber angles is complemented using the main object method. The optimization angles of $\theta_{1}$ and $\theta_{2}$ are $0.8159\left(46^{\circ} 44^{\prime}\right)$ and $-0.8159\left(-46^{\circ} 44^{\prime}\right)$, showed in Table 3 . Both the probability density function and the cumulative distribution function are calculated and compared with the initial results in Fig. 7. The probability density function of the optimization is very tall, thin and intensive compared with the initial probability density function. Besides, its displacement field is overall much less than the initial result, and it indicates that the optimization result performs much better than the initial result in the stiffness character and the capability of resisting the fluctuation in displacement. The result of 
optimized and initial reliability indices based on displacement criteria are provided in Fig. 8. The displacement criterion acts as the boundary to calculate reliability index, and the structure would be in failure when key-point's displacement exceeds the displacement criterion. Once the displacement criteria change, the reliability indices would vary comparatively. The optimized reliability index $\beta$ is much larger than the initial one, which means that the optimization structure is more stable and reliable once the displacement criteria are fixed. The results of Object $_{1}$ and Object $_{2}$ are listed in Table 4, respectively. Obviously, the optimization procedure largely improves the stiffness $\left(\right.$ Object $\left._{2}\right)$ and the displacement stability $\left(\right.$ Object $\left._{1}\right)$ of the key-point. The displacement of key-point becomes much less sensitive to the uncertain parameters of the composite structure. It is the key-feature for composite structure to keep the stable performance in uncertain working conditions (external load). It proves that the optimization procedure is effective, and can obtain a better result.

\subsection{Composite shell with uncertain material parameters and fiber orientations}

The composite Scordelis-Lo roof is a typical shell model and has the potential to apply into the advanced architecture. However, the material uncertainties of composite Scordelis-Lo roof can affect the performance characteristics and even lead to the failure condition. The composite material parameters are usually measured by corresponding experiments and, unavoidably, it causes the geodesic and experimental errors which may present the regularity somehow. It could be described as the probability distribution according to the experiment data. Currently, the material parameters $E_{1}$ and $E_{2}$ are treated as the stochastic variables which are both 
non-Gaussian probabilistic model which can be actually determined by the experiment data. Meanwhile, the manufacturing uncertainty of fiber angle of each ply also affects the result, especially in high precise determination of composite reliable problems. And all the uncertain variables are lised in Table 5, defined as the different probability distributions.

The composite Scordelis-Lo roof is showed in Fig. 9, whose two curved edges are supported by rigid diaphragms and other two edges are free. The geometry and fiber material properties as [35] are listed: radius $R=25$, the length of the $\operatorname{roof} L=50$, the thickness $t=0.25$, Young's modules $E_{11}=3.0 \times 10^{7}, E_{22}=0.75 \times 10^{6}, G_{12}=4.5 \times 10^{5}$, $G_{23}=3.75 \times 10^{5}, G_{13}=4.5 \times 10^{5}, v_{12}=v_{13}=v_{23}=0.25$. The model is subjected to the self-weight $q=-90$ in the z-direction and the angle $\phi$ is $40^{\circ}$. The two-cross-ply shell is symmetrically laminated with $[0,90]$. The reference solution is calculated by SR8 elements of ABAQUS using 14725 nodes in Table 6.

To investigate the accuracy of the present framework, the probability density functions and the cumulative distribution functions respectively obtained from the MCs with 100,000 samples and the present method are plotted in Fig. 10. It indicates that the probability density function of the deflection of node $\mathrm{A}$ is non-Gaussian remarkably. Furthermore, it can be seen that the probability density function and the cumulative distribution function of the node $A$ acquiring by the present method match the reference results well. Then it also illustrates that the accuracy of the present method is sufficient when used as predicting the probability density function and the cumulative distribution function of composite shells with random variables. 
The fiber orientations of optimization are listed in the Table 7. To verify the efficiency of optimization, values of optimization objectives are compared with the initial result in Table 8, and it is obvious that the results have been largely improved in the displacement filed $\left(\right.$ Object $\left._{2}\right)$ and in capability to resist the displacement fluctuation $\left(\right.$ Object $\left._{1}\right)$. For more details about the result, the probability density functions and the cumulative distribution functions of initial and optimization are plotted in Fig. 11.The probability density function of the optimization is thinner and taller, and its displacement field is less than the initial result. It shows that the optimization result performs much better than the initial result in the stiffness character and in the capability to resist the fluctuation of displacement. Furthermore, the optimized and initial reliability indices are plotted in Fig.12, and overall it proves that the optimization procedure is efficient and effective. The framework can perform the prediction and optimization role well for the fiber-reinforced composite structure with the uncertain multi-variables.

\subsection{Composite top cover of automobile with uncertain geometry parameters and}

\section{fiber orientations}

In automobile industry, the fiber-reinforced composite is the important structure applied into cover panel of the automobile. In this practical example, the fiber-reinforced composite shell is designed as the top cover of the automobile showed in Fig. 13. Geometry parameters are not always exact values, especially considering the uncertainty of manufacture and tolerances. It is reasonable to build the probability model to describe this uncertainty according to the experimental data. 
Besides, the lognormal distribution is selected to describe the uncertainty of the important geometry parameter $t$. All the probabilistic models of uncertain parameters are listed in Table. 10.

The geometry and fiber material properties as [35] are listed: thickness $t=0.0$, Young's modules $E_{11}=3.0 \times 10^{7}, E_{22}=0.75 \times 10^{6}, G_{12}=4.5 \times 10^{5}, G_{23}=3.75 \times 10^{5}$, $G_{13}=4.5 \times 10^{5}, \quad v_{12}=v_{13}=v_{23}=0.25$. The model is subjected to the concentrated force $q=-50$ in the middle area in the z-direction. The four-ply shell is symmetrically laminated with [60/-60/60/-60]. To correctly verify the determined solution, the result is compared with the reference solution calculated by S3 elements of ABAQUS using the same number of nodes (494), showed in Table 9.

To testify the accuracy of uncertain analysis using the present method, the probability density function and the cumulative distribution function of the key-point calculated by MCM with 100000 samples and the present method are plotted in Fig. 14. It shows that the probability density function and the cumulative distribution functions of the deflection in key-point calculated by the present method match the reference results well. As a consequence, it is a brilliant method to predict the probability density function and the cumulative distribution function instead of the low-efficient MCs.

The fiber orientation of the initial and optimization are listed in Table 11. In order to prove the efficiency of optimization, the objective values of initial and optimization results are listed in Table 12. It is obvious that Object2 (the displacement criterion) is much less than the initial displacement of key-point and Object1 (the 
displacement fluctuation criterion) is also much smaller than the initial result. Furthermore, the probability density functions and the cumulative distribution functions of initial and optimization are respectively plotted in Fig. (15). The probability density function of the optimization is very taller and thinner compared with the initial probability density function, and its displacement field is much less than the initial result. It indicates that the optimization result performs much better than the initial result in the stiffness character and the capability to resist the fluctuation of displacement. Also, the initial and optimized reliability index of top cover model of the automobile based on the displacement criteria is plotted in Fig. 16, and the optimized reliability index $\beta$ is much larger than the initial one. It means that the optimized structure is more reliable and less likely to fail. Overall, the optimization procedure is efficient and effective, and other objectives, such as stress, can be explored in the same way.

\section{Conclusion}

The integration of performance prediction and optimization for the uncertain fiber-reinforced composite structure based on the transformed perturbation method, the edged-based smoothing technique and the optimization theory is presented. It proves that it is a brilliant method to explicitly predict and optimize the probability density function and the cumulative distribution function instead of the low-efficient MCs. The characteristics of this strategy are summarized as follows:

(1)For the purpose of significantly improving accuracy without increasing the computational cost, the edged-based smoothing technique is introduced into the 
framework. Consequently, it inherits the superior characters, such as the high-efficiency and the capability to resist the mesh distortion. Moreover, it just uses the triangle elements to get highly accurate solutions without costing extra time, which is much more efficient than traditionally stochastic FEM.

(2)Based on the perturbation theory, the non-Gaussian probability density functions and the cumulative distribution functions of stochastic multi-variables (external loads, material parameters, fiber orientations and geometry size) for stochastic static responses of fiber-reinforced composite structure are explicitly exhibited. Unlike the MCs, this strategy only needs one-iteration as the FEM and has the potential in the complex construction. It is more practical and easier to apply into the reliability problem of engineering as the prediction when analytic solution is difficult to obtain.

(3)In optimization procedure, the objectives in the structural stability and maximum stiffness criteria based on the second-order perturbation expansion are proposed, and the overall uncertain condition coupled by uncertain fiber orientation, external load, material parameters and geometry sizes is analyzed and optimized using this framework.

\section{Acknowledgments}

The support of The National Science Foundation of China (11472101) and State Key Program of National Natural Science of China (61232014) are gratefully acknowledged. 


\section{References}

1. T.U. Kim, H.C. Sin, Optimal design of composite laminated plates with the discreteness in ply angles and uncertainty in material properties considered, Comput. Struct. 79(29-30) (2001) 2501-2509.

2. A.K. Onkar, C.S. Upadhyay, D. Yadav, Probabilistic failure of laminated composite plates using the stochastic finite element method, Compos. Struct. 77 (2007) 79-91.

3. A. Vanaerschot, B.N. Cox, S.V. Lomov, D. Vandepitte, Experimentally validated stochastic geometry description for textile composite reinforcements, Compos. Sci. Technol. 122(2016) 122-129.

4. M.F. Ngah, A. Young, Application of the spectral stochastic finite element method for performance prediction of composite structures, Compos. Struct. 78(2007) 447-456.

5. S. Chakraborty, B. Mandal, R. Chowdhury, A. Chakrabarti, Stochastic free vibration analysis of laminated composite plates using polynomial correlated function expansion, Compos. Struct. 135(2016) 236-249.

6. N. Carrere, Y. Rollet, F.-H. Leroy, J.-F. Maire, Efficient structural computations with parameters uncertainty for composite applications, Compos. Sci. Technol. 69(2009) $1328-1333$.

7. G. Stefanou, D. Savvas, M. Papadrakakis, Stochastic finite element analysis of composite structures based on material microstructure, Compos. Struct. 132 (2015) 384-392.

8. M.M.S. Dwaikat, C. Spitas, V. Spitas, Effect of the stochastic nature of the constituents parameters on the predictability of the elastic properties of fibrous nano-composites, Compos. Sci. Technol. 72 (2012) 1882-1891.

9. T.S. Mesogitis, A.A. Skordos, A.C. Long, Stochastic simulation of the influence of cure kinetics uncertainty on composites cure, Compos. Sci. Technol. 110 (2015) 145-151.

10. K. Sepahvand, Spectral stochastic finite element vibration analysis of fiber-reinforced composites with random fiber orientation, Compos. Struct. 145(2016) 119-128.

11. B.Z Xia, D.J Yu, J. Liu, Transformed perturbation stochastic finite element method for static response analysis of stochastic structure, Finite Elem. Anal. Des. 79(2014) 9-21.

12. O. Cavdar, A. Bayraktar, A. Cavdar, S. Adanur, Perturbation based stochastic finite element analysis of the structural systems with composite sections under earthquake forces, Steel Compos. Struct. 8(2008) 129-144.

13. M. Kamiński, On stochastic finite element method for linear elastostatics by the Taylor expansion, Struct. Multidisc. Optim. 35(2008) 213-223.

14. B. Rong, X.T. Rui, L. Tao, Perturbation finite element transfer matrix method for random eigenvalue problems of uncertain structures, J. Appl. Mech. 79( 2012) 021005.

15. H.M. Panayirci, G.I. Schuëller, On the capabilities of the polynomial chaos expansion method within SFE analysis - an overview, Arch. Comput. Methods Eng. 18 (2011) 43-55.

16. S. Adhikari, Doubly spectral stochastic finite - element method for linear structural dynamics, J. Aerosp. Eng. 24 (2011) 264-276.

17. M. Kleiber, T.D. Hien, The Stochastic Finite Element Method, John Wiley \&Sons, New York, 1992.

18. G.I. Schuëller, Developments in stochastic structural mechanics, Arch. Appl. Mech. 75 (2006) 755-773. 
19. G. Stefanou, The stochastic finite element method: past, present and future,Comput. Methods Appl. Mech. Eng. 198 (2009) 1031-1051.

20. C. Papadimitriou, L.S. Katafygiotis, J.L. Beck, Approximate analysis of response variability of uncertain linear systems, Prob. Eng. Mech. 10 (1995) 251-264.

21. M.M. Kaminski, M.T. Strakowski, On the least squares stochastic finite element analysis of the steel skeletal towers exposed to fire, Arch. Civ. Mech. Eng. 13 (2013) 242-253.

22. M. Kaminski, On the dual iterative stochastic perturbation-based finite-element method in solid methcanics with Gaussian uncertainties, Internat. J. Numer. Methods Engrg. 104 (2015) 1038-1060.

23. J.Q. Li, X.P. Tian, Z.J. Han, Y. Narita, Stochastic thermal buckling analysis of laminated plates using perturbation technique, Compos. Struct. 139 (2016) 1-12.

24. M. Talha, B.N. Singh, Stochastic perturbation-based finite element for buckling statistics of FGM plates with uncertain material properties in thermal environments, Compos. Struct. 108(2014) 823-833.

25. A. Keese, H.G. Matthies, Hierarchical parallelization for the solution of stochastic finite element equations, Comput. Struct. 83(2005) 1033-1047.

26. M. Kamiński, Generalized perturbation-based stochastic finite element method in elastostatics, Comput. Struct. 85(2007) 586-584.

27. M. Kaminski, Generalized stochastic perturbation technique in engineering computations, Math. Comput. Modelling. 51 (2010) 272-285.

28. G.R. Liu, X. Zhao, K.Y. Dai, Z.H. Zhong, G.Y. Li, X. Han, Static and free vibration analysis of laminated composite plates using the conforming radial point interpolation method, Compos. Sci. Technol. 68 (2008) 354-366.

29. G.R. Liu, W. Zeng, H. Nguyen-Xuan, Generalized stochastic cell-based smoothed finite element method (GS_CS-FEM) for solid mechanics, Finite Elem Anal Des. 63(2013) 51-61.

30. G.R. Liu, T.T. Nguyen, K.Y. Lam, An edge-based smoothed finite element method (ES-FEM) for static and dynamic problems of solid mechanics, J. Sound Vib. 320 (2009) 1100-1130.

31. Z.C. He, G.R. Liu, Z.H. Zhong, G.Y. Zhang, A.G. Cheng, A coupled ES-FEM/BEM method for fluid-structure interaction problems, Eng.Anal. Bound. Elem. 35 (1) (2011) 140-147.

32. L. Chen, G.R. Liu, N. Nourbakhsh-Nia, K. Zeng, A singular edge-based smoothed finite element method (ES-FEM) for bimaterial interface cracks, Comput. Mech. 45 (2-3) (2010) $109-125$.

33. G.R. Liu, N. Nourbakhshnia, Y.W. Zhang, A novel singular ES-FEM method for simulating singular stress fields near the crack tips for linear fracture problems, Eng. Fract. Mech. 78 (6) (2011) 863-876.

34. Z.C. He, G.R. Liu, Z.H. Zhong, S.C. Wu, G.Y. Zhang, A.G. Cheng, An edge-based smoothed finite element method (ES-FEM) for analyzing three-dimensional acoustic problems, Comput. Methods Appl. Mech. Engrg. 199 (1) (2009) 20-33.

35. X.Y. Cui, G.R. Liu, G.Y. Li, Bending and vibration of laminated composite plates using an edge-based smoothing technique, Eng.Anal. Bound. Elem. 35(2011) 818-826.

36. H. Wang, Y. Zeng, E.Y. Li, G.X. Huang, G.Q. Gao, G.Y. Li, "Seen Is Solution” a CAD/CAE integrated parallel reanalysis design system, Comput. Methods Appl. Mech. Engrg. 299 (2016) 187-214.

37. P. Pedersen, On thickness and orientational design with orthotropic materials, Struct. 
Optimiz. 3(1991) 69-78.

38. J.H. Luo, H.C. Gea, Optimal orientation of orthotropic material using an energy based method, Struct. Optimiz. 15(1998) 230-236.

39. P. Pedersen, On optimal orientation of orthotropic materials, Struct. Optimiz. 1(1989) 101-106.

40. C.W. Kim, W. Hwang, H.C. Park, K.S. Han, Stacking sequence optimization of laminated plates, Compos. Struct. 39(1997) 283-288.

41. W.J. Park, An optimal design of simple symmetric laminates under the first ply failure criterion, J. Compos. Mater. 16(1982) 341-355.

42. T.Y. Kam, R.R. Chang, Optimum layup of thick laminated composite plates for maximum stiffness, Eng. Optimiz. 19(1992) 237-49.

43. T.R. Tauchert, S. Adibhalta, Design of laminated plates for maximum stiffness, J. Compos. Mater. 18(1984) 58-69.

44. T.Y. Kam, M.D. Lai, Multilevel optimal design of laminated composite plate structure. Compos. Struct. 31(1989)197-202.

45. V.M.Franco Correia, C.M.Mota Soares, C.A.Mota Soares, Higher order models on the eigenfrequency analysis and optimal design of laminated composite structures, Compos. Struct. 39(1997) 237-53.

46. C.M.Mota Soares, C.A.Mota Soares, V.M.Franco Correia, Optimization of multiaminated structures using higher order deformation models. Comput. Methods Appl. Mech. Eng. 149(1997) 133-52.

47. C. Jiang, X. Han, G.P. Liu, Uncertain optimization of composite laminated plates using a nonlinear interval number programming method, Comput. Struct. 86(2008) 1696-1703.

48. M. Rouhi, M. Rais-Rohani, Modeling and probabilistic design optimization of a nanofiber-enhanced composite cylinder for buckling, Compos. Struct. 95(2013) 346-353.

49. M. Rouhi, M. Rais-Rohani, A. Najafi, Probabilistic analysis and optimization of energy absorbing components made of nanofiber enhanced composite materials, Compos. Struct. 100(2013) 144-153.

50. K.U. Bletzinger, M. Bischoff, E. Ramm, A unified approach for shear-locking-free triangular and rectangular shell finite elements, Comput. Struct. 75 (2000) 321-34. 


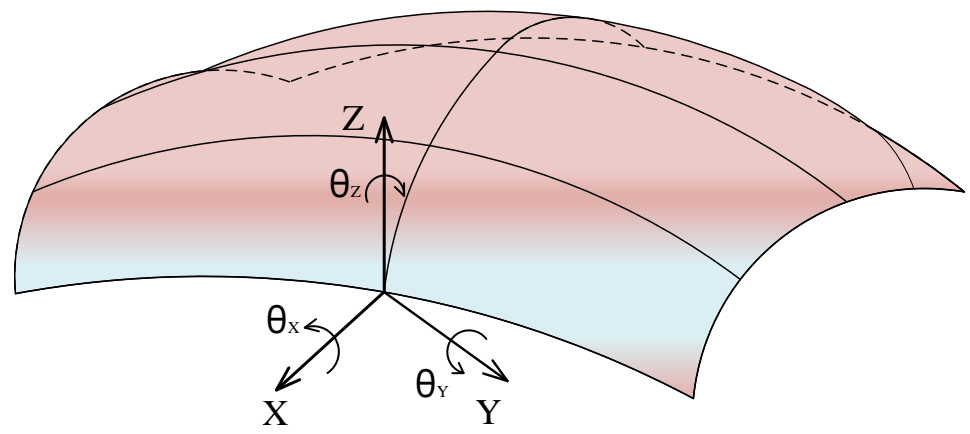

Fig. 1. The typical shell model and its global coordinates.

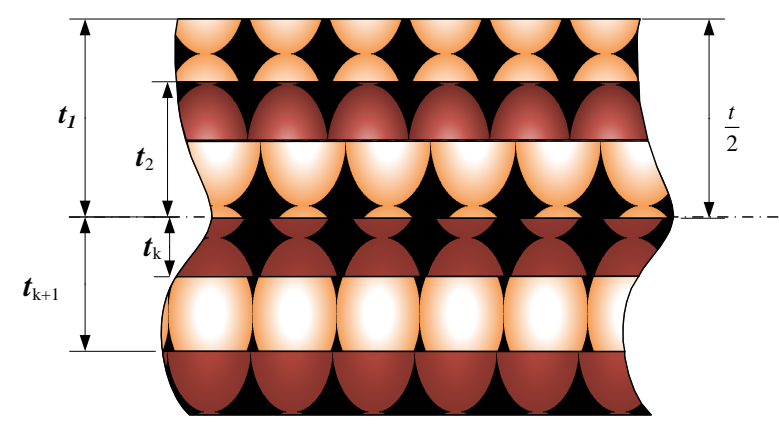

Fig. 2. Cross-sectional view of the laminated plate.
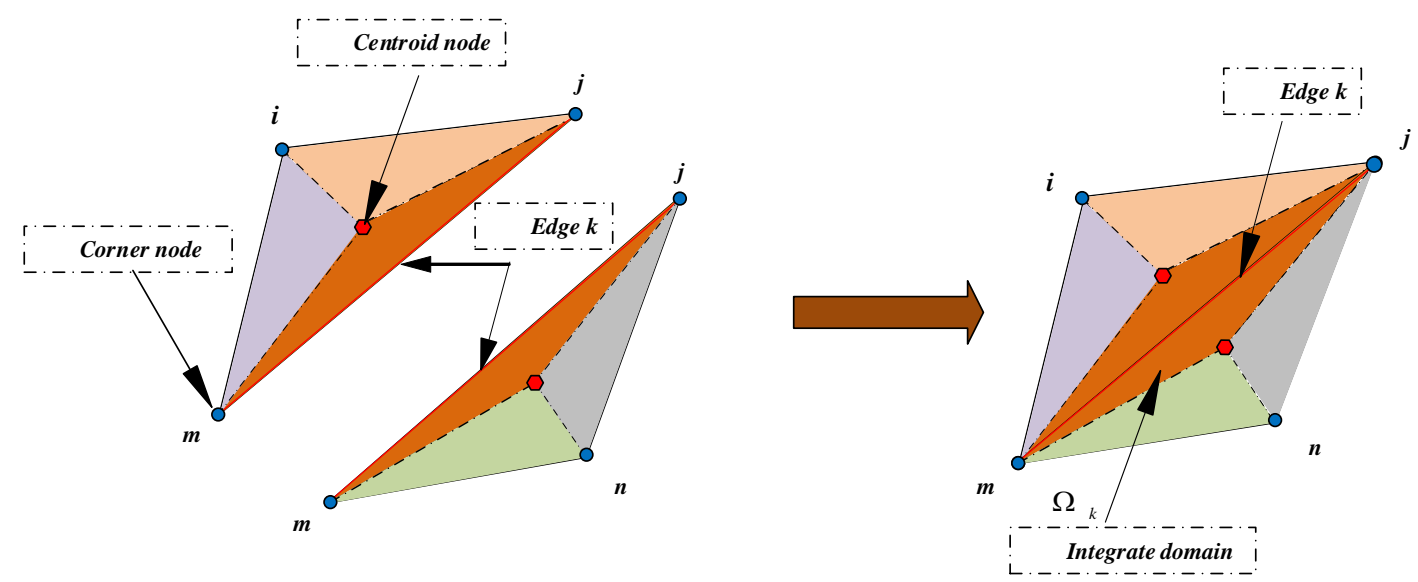

Fig. 3. The integration domain of $\Omega_{k}$ of internal edge $k$. 


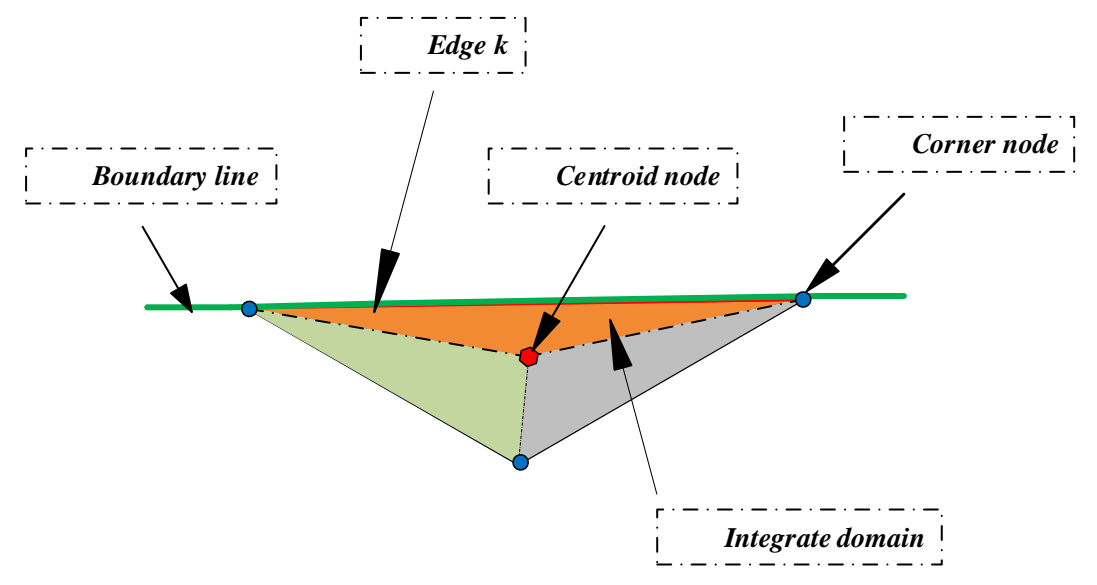

Fig. 4. The integration domain of boundary edge $k$.

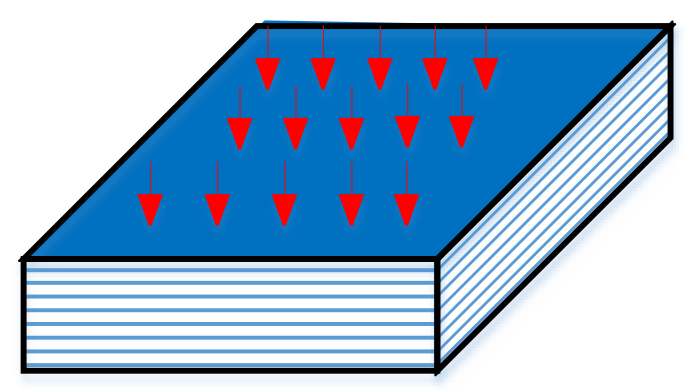

(a)

Key-point $\bigcirc$ Corner-point

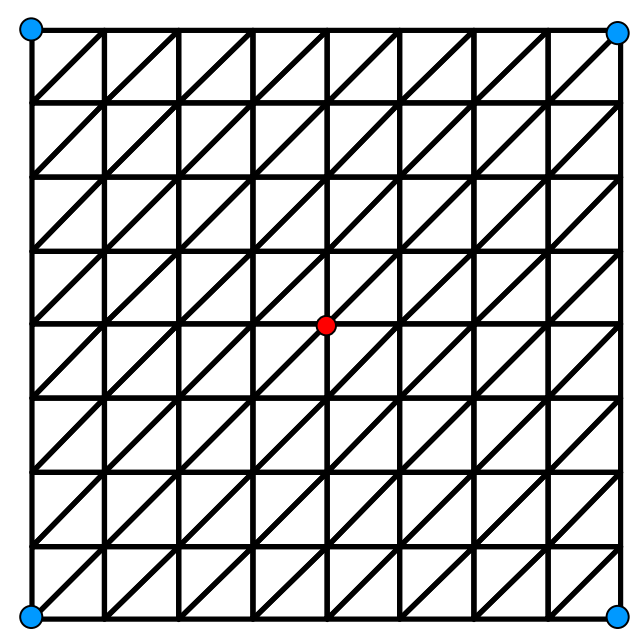

(b)

Fig. 5. The composite plate subjecting to the uniform surface moment. 


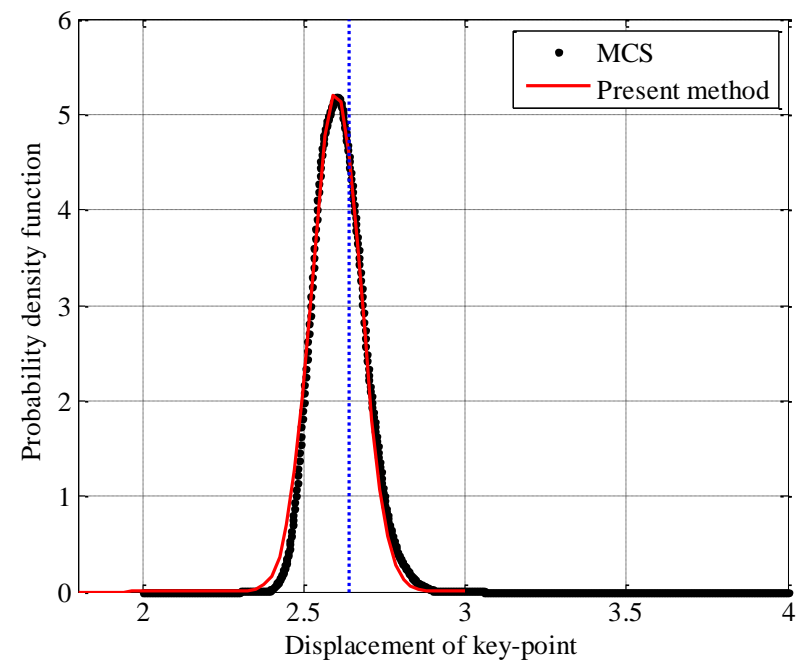

(a)

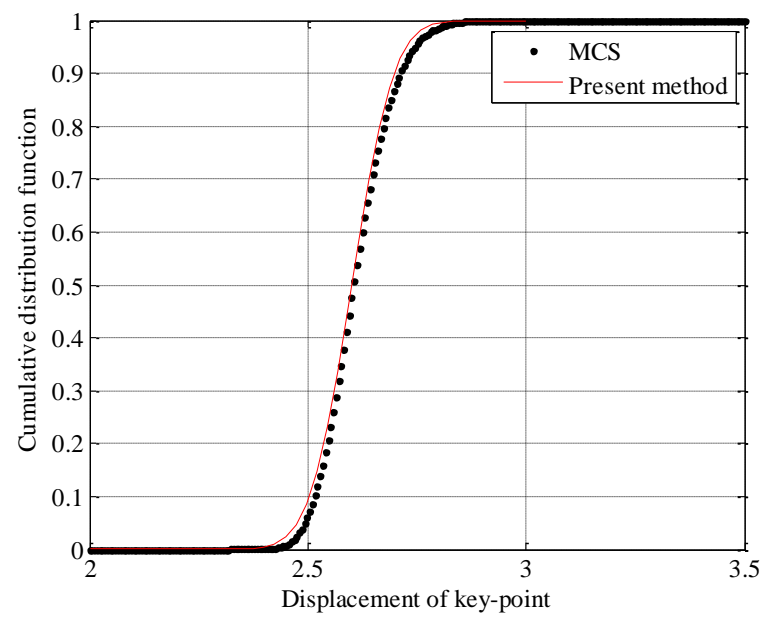

(b)

Fig. 6. The probability density functions and cumulative distribution functions of the deflection of the key-point calculated by MCs and TPSEM. 


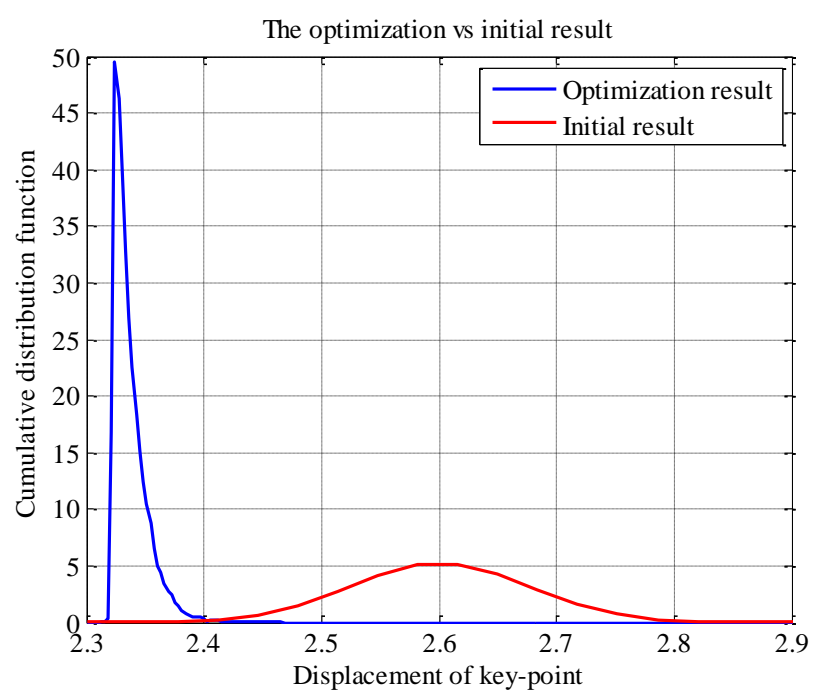

(a)

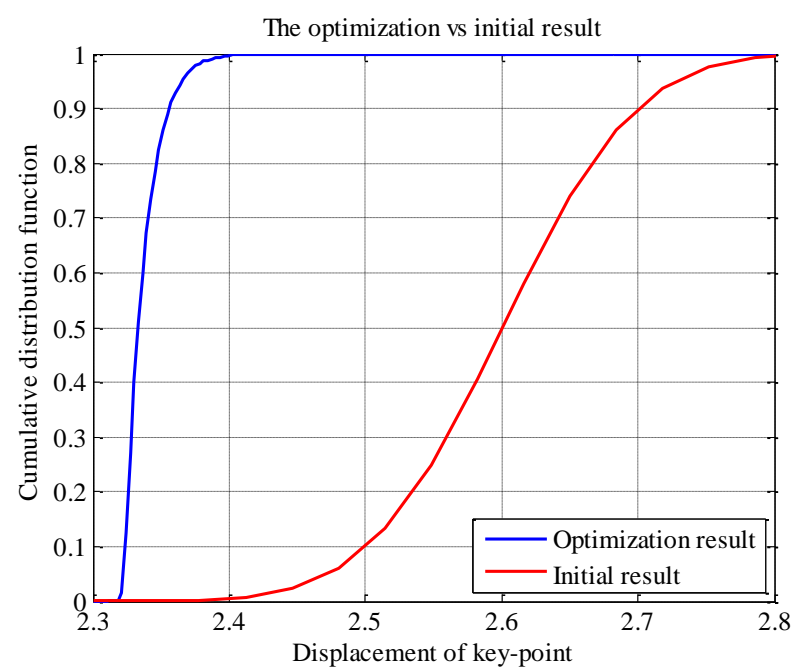

(b)

Fig. 7. The probability density functions and cumulative distribution functions of the deflection of the key-point are calculated based on the initial and optimization fiber orientation. 


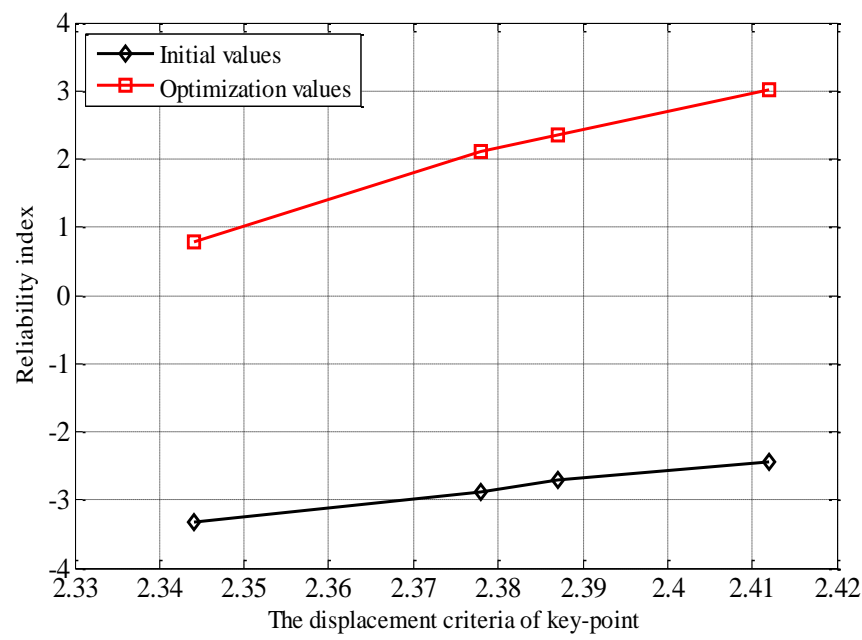

Fig. 8. The initial and optimized reliability index of composite plate based on the displacement criteria.

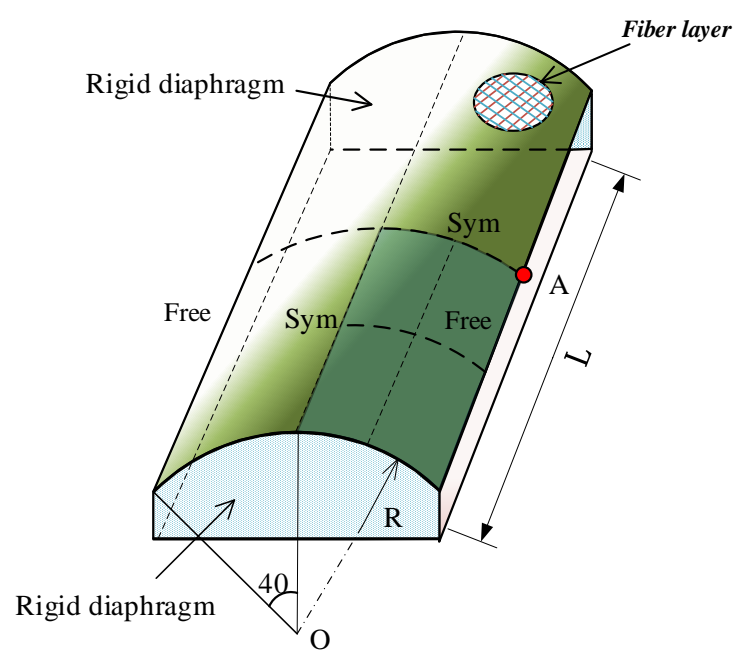

Fig. 9. The composite Scordelis-Lo roof model subjected to the self-weight. 


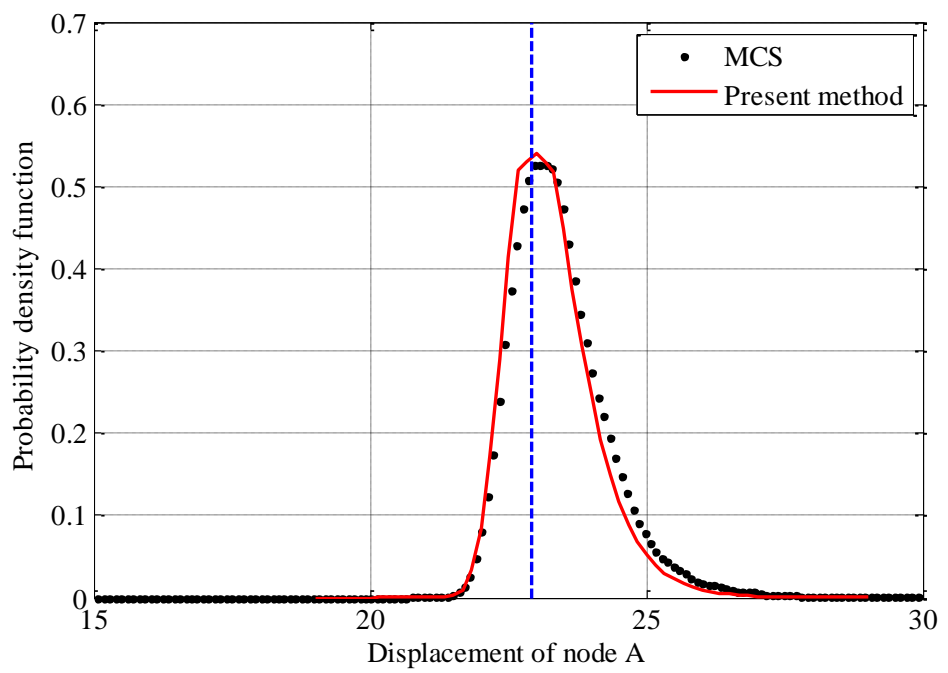

(a)

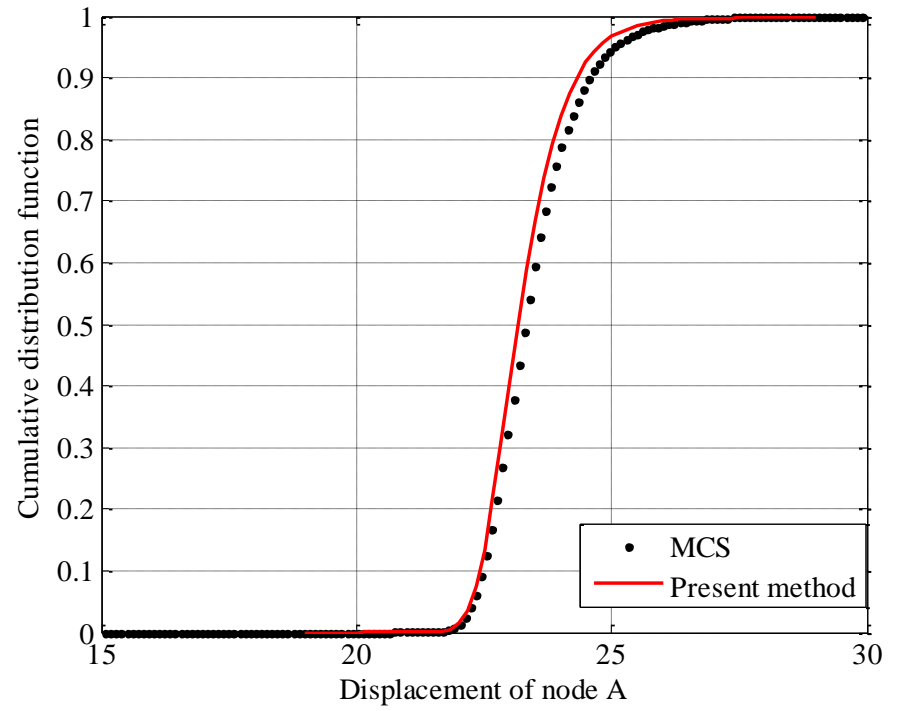

(b)

Fig. 10. The probability density functions and cumulative distribution functions of the deflection of the key-point calculated by MCs and TPSEM. 


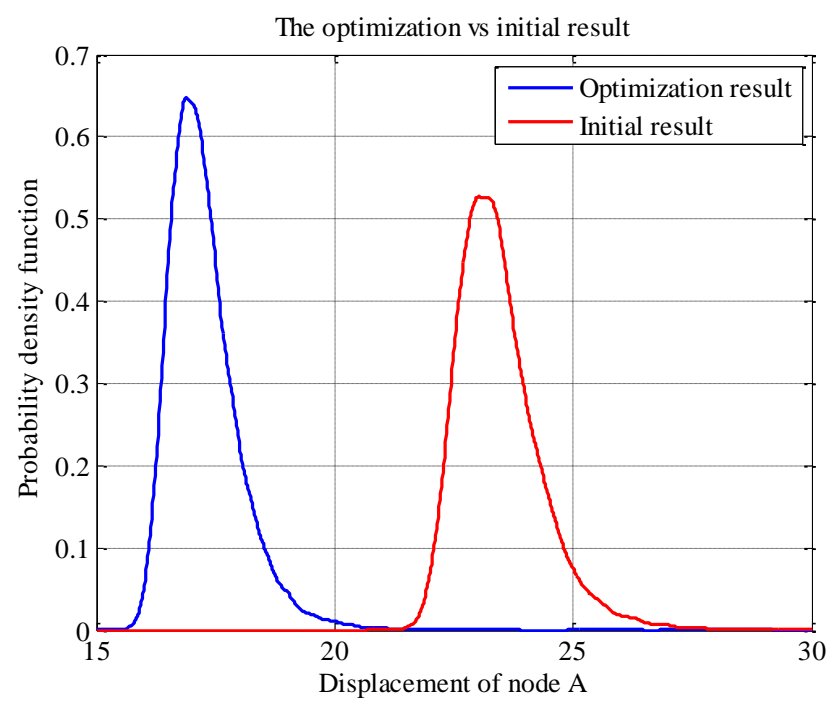

(a)

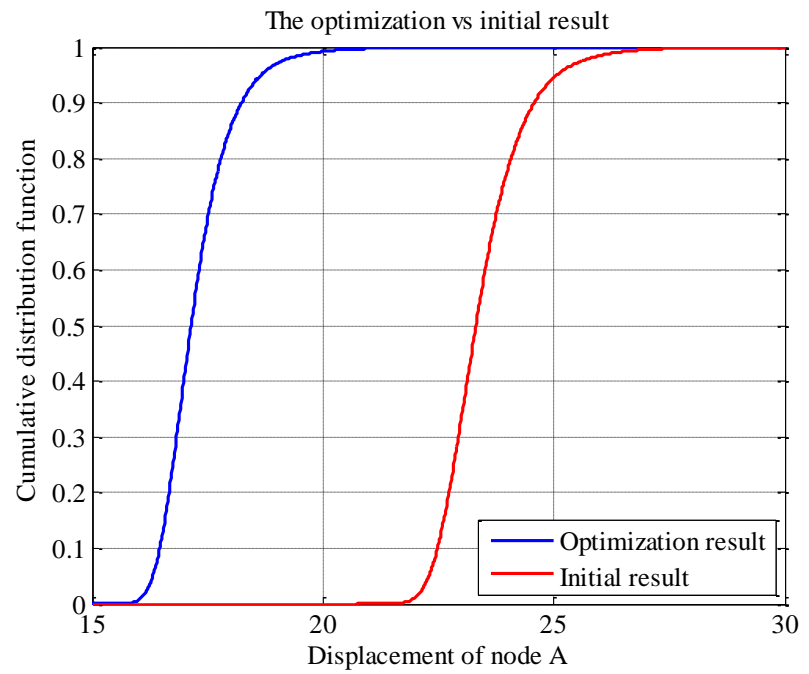

(b)

Fig. 11. The probability density functions and cumulative distribution functions of the deflection of the key-point are calculated based on the initial and optimization fiber orientation. 


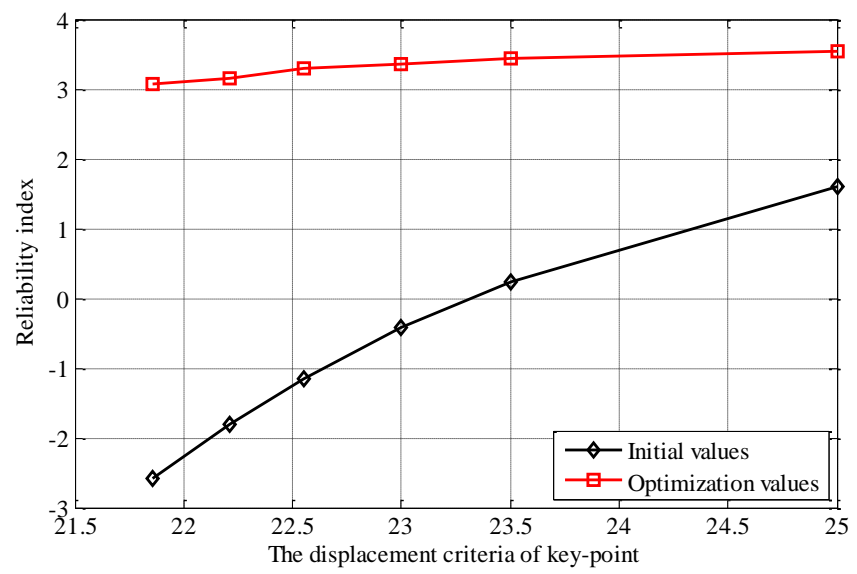

Fig. 12. The initial and optimized reliability index of composite Scordelis-Lo roof model based on the displacement criteria.

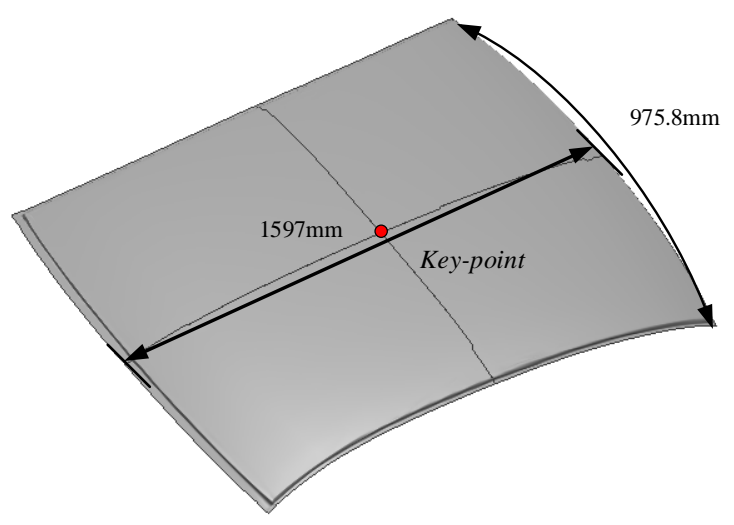

Fig. 13. The top cover model of the automobile. 


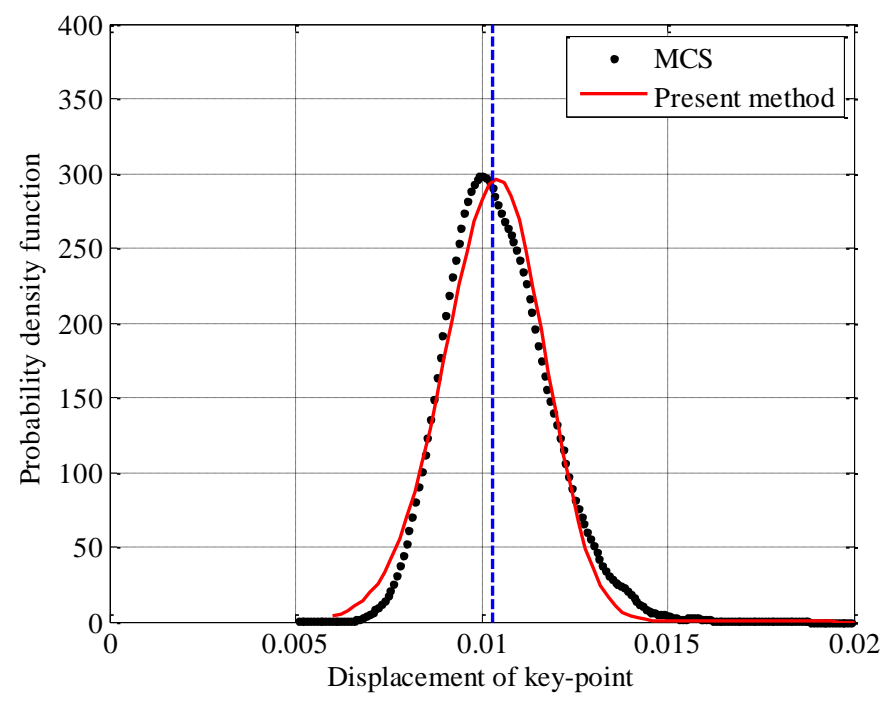

(a)

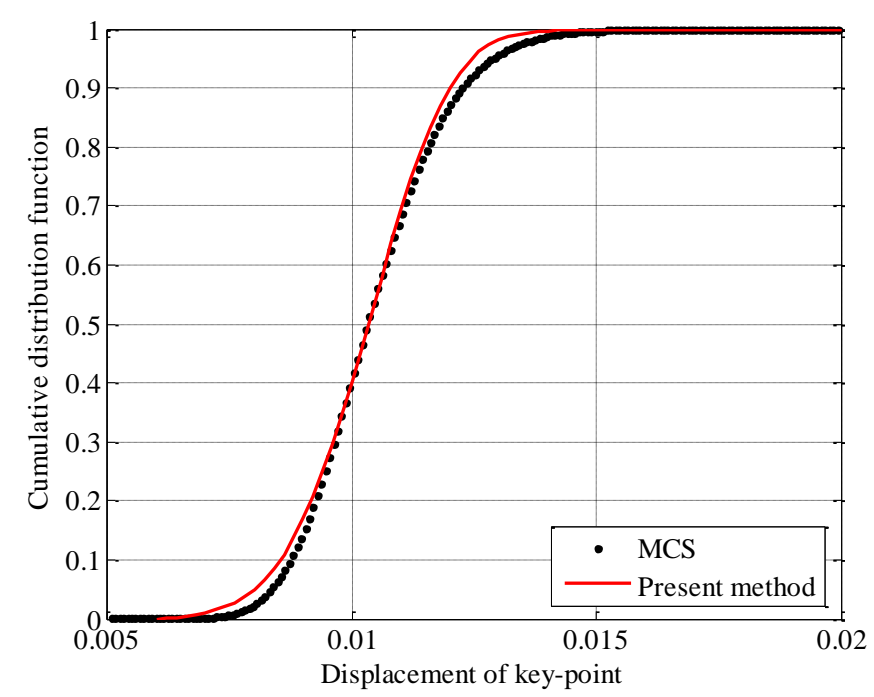

(b)

Fig. 14. The probability density functions and cumulative distribution functions of the deflection of the key-point calculated by MCs and TPSEM. 


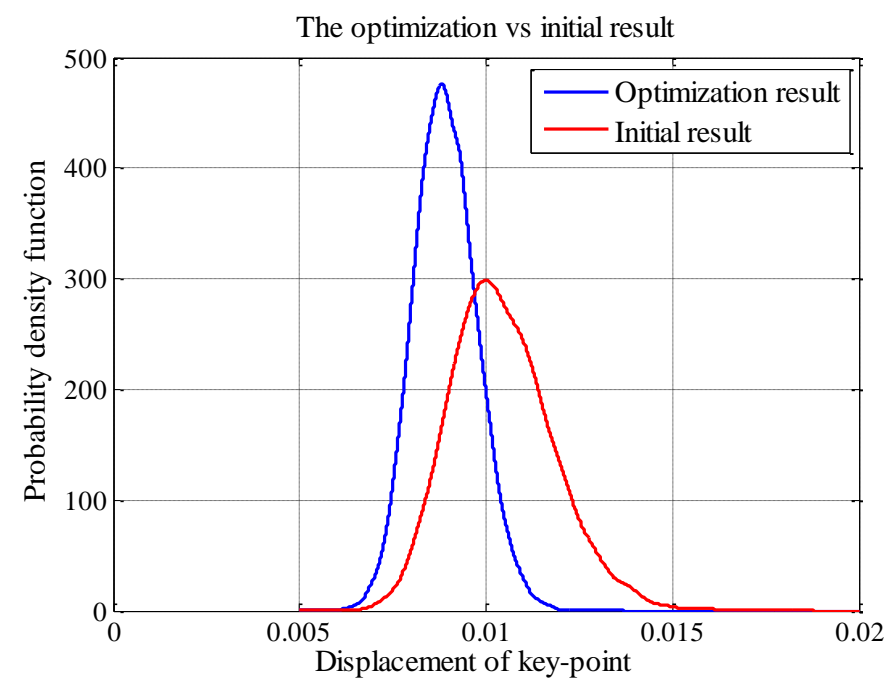

(a)

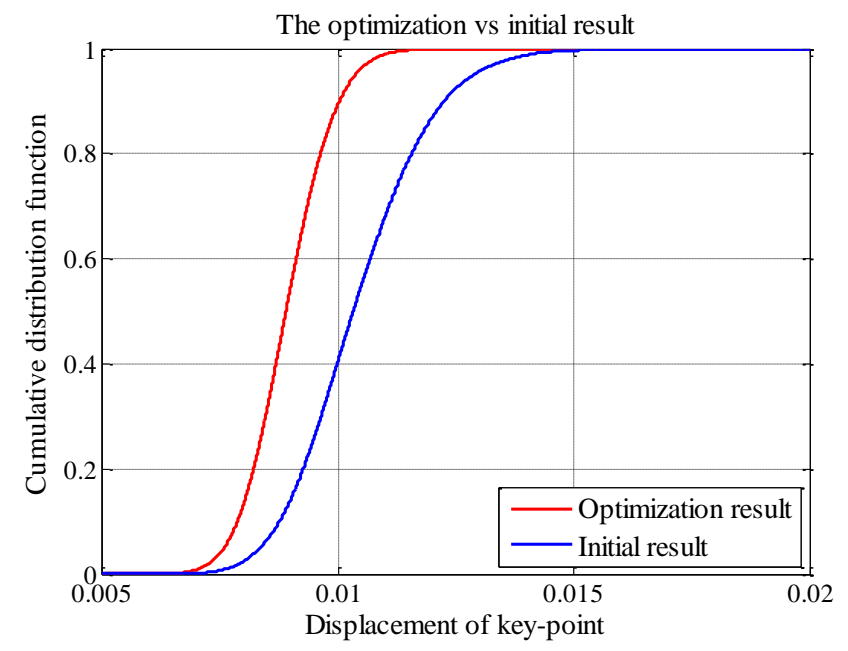

(b)

Fig. 15. The probability density functions and cumulative distribution functions of the deflection of the key-point are calculated based on the initial and optimization fiber orientation. 


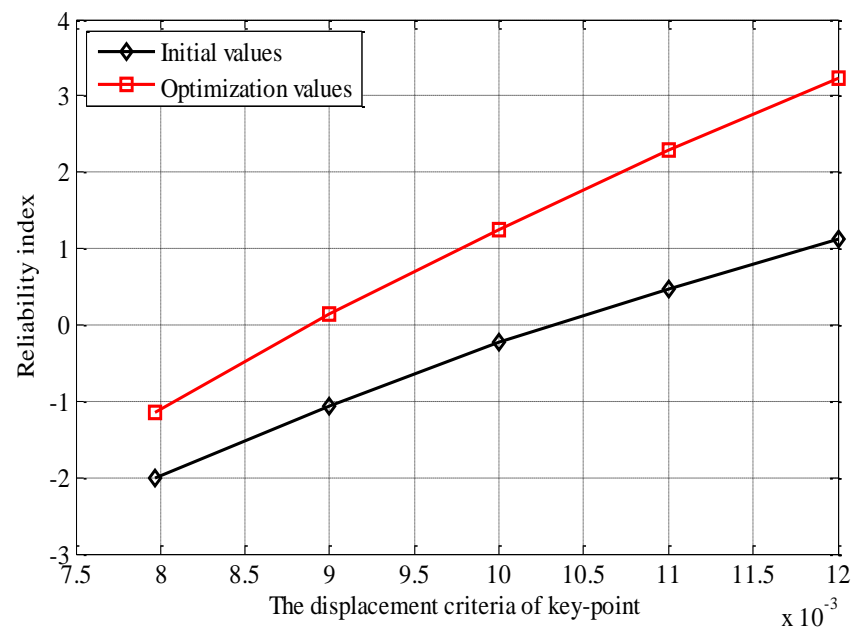

Fig. 16. The initial and optimized reliability index of top cover model of the automobile based on the displacement criteria. 
Table 1. The stochastic variables of external load and fiber orientation.

\begin{tabular}{ccc}
\hline Variables & Distribution & Stochastic parameter of the variables \\
\hline $\mathrm{P}$ & Gumbel (evpdf) & $\mu=100, \sigma=40$ \\
$\theta_{1}$ & Gaussian & $\mu=\mathrm{pi} / 3, \sigma=0.05$ \\
$\theta_{2}$ & Gaussian & $\mu=-\mathrm{pi} / 3, \sigma=0.05$ \\
\hline
\end{tabular}

Table 2. The determination results compared with the Ref.

\begin{tabular}{ccccc}
\hline Point & 81 nodes $(\%)$ & 121 nodes $(\%)$ & 169 nodes $(\%)$ & Ref.(mm) \\
\hline Key-Point & 2.639 & 2.6571 & 2.6674 & 2.694 \\
Error & -2.04 & -1.36 & -0.987 & $/$ \\
\hline
\end{tabular}

Table 3. The fiber orientation results of the initial and optimization.

\begin{tabular}{ccc}
\hline Condition & Initial & Optimization \\
\hline layer & 9 & 9 \\
$\theta_{1}$ & $\mathrm{pi} / 3$ & 0.8159 \\
$\theta_{2}$ & $-\mathrm{pi} / 3$ & -0.8159 \\
\hline
\end{tabular}

Table 4. The objective values of the initial and optimization.

\begin{tabular}{ccc}
\hline Condition & Initial & Optimization \\
\hline Object $_{1}$ & 0.00504502 & $6.4789 \mathrm{e}-5$ \\
Object 2 & 2.6387 & 2.25047 \\
\hline
\end{tabular}

Table 5. The stochastic variables of material parameters and fiber orientation.

\begin{tabular}{ccc}
\hline Variables & Distribution & Stochastic parameter of the variables \\
\hline $\mathrm{E}_{1}$ & Gumbel (evpdf) & $\mu=3.0 \mathrm{e} 7, \sigma=1 \mathrm{e} 6$ \\
$\mathrm{E}_{2}$ & Gumbel (evpdf) & $\mu=0.75 \mathrm{e} 6, \sigma=0.5 \mathrm{e} 5$ \\
$\theta_{1}$ & Gaussian & $\mu=\mathrm{pi} / 2, \sigma=0.02$ \\
$\theta_{2}$ & Gaussian & $\mu=0, \sigma=0.02$ \\
\hline
\end{tabular}

Table 6. The determination results compared with the Ref.

\begin{tabular}{ccc}
\hline Point & 961 nodes & Ref.(14725nodes) \\
\hline Key-Point & 25.42 & 28.20 \\
Percent $(\%)$ & $90.14 \%$ & $/$ \\
\hline
\end{tabular}


Table 7. The fiber orientation results of the initial and optimization.

\begin{tabular}{ccc}
\hline Condition & Initial & Optimization \\
\hline layer & 2 & 2 \\
$\theta_{1}$ & $\mathrm{pi} / 2$ & -0.062831 \\
$\theta_{2}$ & 0 & -0.125664 \\
\hline
\end{tabular}

Table 8. The objective values of the initial and optimization.

\begin{tabular}{ccc}
\hline Condition & Initial & Optimization \\
\hline Object $_{1}$ & 0.37667 & 0.0816696 \\
Object $_{2}$ & 22.9367 & 16.761779 \\
\hline
\end{tabular}

Table 9. The determination results compared with the Ref.

\begin{tabular}{ccc}
\hline Point & 961 nodes & Ref. \\
\hline Key-Point & $-1.0067 \mathrm{e}-2$ & $-1.0193 \mathrm{e}-2$ \\
Error $(\%)$ & $-1.23 \%$ & $/$ \\
\hline
\end{tabular}

Table 10. The stochastic variables of shell thickness and fiber orientation.

\begin{tabular}{ccc}
\hline Variables & Distribution & Stochastic parameter of the variables \\
\hline $\mathrm{t}$ & lognormal & $\mu=1.884, \sigma=0.06579$ \\
$\theta_{1}$ & Gaussian & $\mu=\mathrm{pi} / 3, \sigma=0.05$ \\
$\theta_{2}$ & Gaussian & $\mu=-\mathrm{pi} / 3, \sigma=0.05$ \\
\hline
\end{tabular}

Table 11. The objective values of the initial and optimization.

\begin{tabular}{ccc}
\hline Condition & Initial & Optimization \\
\hline Object1 & $1.45592 \mathrm{e}-004$ & $6.708489 \mathrm{e}-5$ \\
Object 2 & 0.01027682 & 0.00883552 \\
\hline
\end{tabular}

Table 12. The fiber orientation results of the initial and optimization.

\begin{tabular}{ccc}
\hline Condition & Initial & Optimization \\
\hline Layer & 4 & 4 \\
$\theta_{1}$ & $\mathrm{pi} / 3$ & $\mathrm{pi} / 2$ \\
$\theta_{2}$ & $-\mathrm{pi} / 3$ & $-\mathrm{pi} / 2$ \\
\hline
\end{tabular}

